\title{
Description and evaluation of the Model for Ozone and Related chemical Tracers, version 4 (MOZART-4)
}

\author{
L. K. Emmons ${ }^{1}$, S. Walters ${ }^{1}$, P. G. Hess ${ }^{1, *}$, J.-F. Lamarque ${ }^{1}$, G. G. Pfister ${ }^{1}$, D. Fillmore ${ }^{1, * *}$, C. Granier ${ }^{2,3}$, \\ A. Guenther ${ }^{1}$, D. Kinnison ${ }^{1}$, T. Laepple ${ }^{1, * * *}$, J. Orlando ${ }^{1}$, X. Tie ${ }^{1}$, G. Tyndall ${ }^{1}$, C. Wiedinmyer ${ }^{1}$, S. L. Baughcum ${ }^{4}$, and \\ S. Kloster ${ }^{5, *}$ \\ ${ }^{1}$ National Center for Atmospheric Research, Boulder, CO, USA \\ ${ }^{2}$ Centre National de la Recherche Scientifique, Paris, France \\ ${ }^{3}$ NOAA, Earth System Research Laboratory, Boulder, CO, USA \\ ${ }^{4}$ Boeing Company, Seattle, WA, USA \\ ${ }^{5}$ Max-Planck-Institute for Meteorology, Hamburg, Germany \\ *now at: Department of Biological and Environmental Engineering, Cornell University, Ithaca, NY, USA \\ *** now at: Tech-X Corporation, Boulder, CO, USA \\ ${ }^{* * *}$ now at: Alfred Wegener Institute for Polar and Marine Research, Bremerhaven, Germany
}

Received: 6 August 2009 - Published in Geosci. Model Dev. Discuss.: 27 August 2009

Revised: 7 December 2009 - Accepted: 8 December 2009 - Published: 12 January 2010

\begin{abstract}
The Model for Ozone and Related chemical Tracers, version 4 (MOZART-4) is an offline global chemical transport model particularly suited for studies of the troposphere. The updates of the model from its previous version MOZART-2 are described, including an expansion of the chemical mechanism to include more detailed hydrocarbon chemistry and bulk aerosols. Online calculations of a number of processes, such as dry deposition, emissions of isoprene and monoterpenes and photolysis frequencies, are now included. Results from an eight-year simulation (2000-2007) are presented and evaluated. The MOZART- 4 source code and standard input files are available for download from the NCAR Community Data Portal (http://cdp.ucar.edu).
\end{abstract}

\section{Introduction}

MOZART-4 (Model for Ozone and Related chemical Tracers, version 4) is a global chemical transport model for the troposphere and includes a number of updates over the previous tropospheric version MOZART-2 (Horowitz et al., 2003). MOZART-3 is an extension of MOZART-2 with detailed stratospheric chemistry (Kinnison et al., 2007). The MOZART models are offline models, requiring meteorological fields from either climate models or assimilations of me-

Correspondence to: L. Emmons (emmons@ucar.edu) teorological observations. MOZART is built on the framework of the Model of Atmospheric Transport and Chemistry (MATCH) (Rasch et al., 1997). Convective mass fluxes are diagnosed by the model, using the shallow and mid-level convective transport formulation of Hack (1994) and deep convection scheme of Zhang and MacFarlane (1995). Vertical diffusion within the boundary layer is based on the parameterization of Holstlag and Boville (1993). Wet deposition is taken from the formulation of Brasseur et al. (1998). Advective transport incorporates the flux form semiLagrangian transport algorithm of Lin and Rood (1996), as described in Horowitz et al. (2003). These physical processes have not been updated from MOZART-2. Details of the chemical solver numerics are given in the Auxiliary Material of Kinnison et al. (2007).

MOZART- 4 can be driven by essentially any meteorological data set and with any emissions inventory, so there is not a unique standard simulation. The meteorological variables needed to run MOZART-4 are given in Table 1. Whereas the published evaluation of MOZART-2 was for a simulation driven by climate model meteorology (Horowitz et al., 2003), this work presents MOZART-4 driven by meteorological analyses. A MOZART-4 simulation of 2000-2007 is used here to illustrate components of the model and for evaluation. The model was driven by meteorology from the NCAR reanalysis of the National Centers for Environmental Prediction (NCEP) forecasts (Kalnay et al., 1996; Kistler et al., 2001), at a horizontal resolution of approximately $2.8^{\circ} \times 2.8^{\circ}$,

Published by Copernicus Publications on behalf of the European Geosciences Union. 
Table 1. Meteorological inputs required for MOZART-4.

\begin{tabular}{|c|c|c|c|}
\hline Variable & $\begin{array}{l}\text { MOZART-4 } \\
\text { Name }\end{array}$ & $\begin{array}{l}\text { Dimen- } \\
\text { sion }\end{array}$ & Units \\
\hline Zonal Winds & $\mathrm{U}$ & $3-\mathrm{D}$ & $\mathrm{ms}^{-1}$ \\
\hline Meridional Winds & $\mathrm{V}$ & $3-\mathrm{D}$ & $\mathrm{ms}^{-1}$ \\
\hline Temperature & $\mathrm{T}$ & $3-\mathrm{D}$ & $\mathrm{K}$ \\
\hline Specific Humidity (optional) & Q & $3-\mathrm{D}$ & $\mathrm{kg} \mathrm{kg}^{-1}$ \\
\hline Surface Temperature & TS & 2-D & $\mathrm{K}$ \\
\hline Surface Pressure & PS & $2-\mathrm{D}$ & $\mathrm{Pa}$ \\
\hline Land/Ocean/Sea Ice Flag & ORO & 2-D & unitless \\
\hline Surface Geopotential Height & PHIS & 2-D & $\mathrm{m}^{2} \mathrm{~s}^{-2}$ \\
\hline Surface Zonal Stress & TAUX & $2-\mathrm{D}$ & $\mathrm{Nm}^{-2}$ \\
\hline Surface Meridional Stress & TAUY & 2-D & $\mathrm{Nm}^{-2}$ \\
\hline Surface Heat Flux & SHFLX & 2-D & $\mathrm{W} \mathrm{m}^{-2}$ \\
\hline Surface Moisture Flux & QFLX & 2-D & $\mathrm{kg} \mathrm{m}^{-2} \mathrm{~s}^{-1}$ \\
\hline Solar Flux at Surface & FSDS & $2-\mathrm{D}$ & $\mathrm{W} \mathrm{m}^{-2}$ \\
\hline Snow Height & SNOWH & $2-\mathrm{D}$ & $\mathrm{m}$ \\
\hline Soil Moisture Fraction ${ }^{1}$ & SOILW & 2-D & unitless \\
\hline Previous month's average $\mathrm{TS}^{1}$ & TS_avg & 2-D & $\mathrm{K}$ \\
\hline Previous month's average FSDS ${ }^{1}$ & FSDS_avg & 2-D & $\mathrm{W} \mathrm{m}^{-2}$ \\
\hline
\end{tabular}

${ }^{1}$ If SOILW, TS_avg or FSDS_avg are not available in met fields, a climatology is used.

with 28 vertical levels from the surface to approximately $2 \mathrm{hPa}$. This resolution is typically used for multi-year simulations. MOZART-4 can be run at essentially any resolution, depending on memory limitations, and typically matching the input meteorological fields. Currently the highest resolution that has been run with full chemistry is $0.7^{\circ} \times 0.7^{\circ}$ (Emmons et al., 2010).

MOZART-4 has been tested on computing platforms ranging from a single CPU on a desktop machine to more than one hundred CPUs on a state-of-the-art supercomputer. The following are required for running MOZART-4: the Linux or Unix operating system (including Apple OS X), the cpp utility (available on any Unix or Linux system), the gnu makefile utility, the netcdf utility, and a Fortran 90 compiler. The code has been parallelized using both OpenMP and MPI approaches, with a hybrid MPI-OMP configuration generally giving the fastest runtimes. For desktop systems, MOZART-4 has been compiled and is configured to use either the Portland Group, Lahey/Fujitsu, or Intel Fortran 90 or 95 compilers. All compilers produce similar execution times with normal optimizations. MOZART-4, configured at the standard horizontal resolution of $128 \times 64$ grid boxes with 28 vertical levels, requires at least $2 \mathrm{~GB}$ main memory per cpu, and can be run on single-, dual-, quad- or 8CPU workstations. The source code is available for download from the NCAR Community Data Portal (http://cdp. ucar.edu), after registration and approval from the authors. Instructions on the building and running of MOZART-4 are included with the source code. Further information is posted on the MOZART-4 website (http://gctm.acd.ucar.edu/ mozart/models/m4/index.shtml).
This paper presents a description of MOZART-4, providing details of the features of the model, and serving as a reference for past and future studies using this model. The following section describes the model updates included in MOZART-4 over MOZART-2. Section 3 summarizes previous applications of MOZART-4. The final sections give a description of the 2000-2007 model simulation presented here, and its evaluation through comparison to global networks and satellite observations.

\section{Updates to model processes}

Details of the improvements to MOZART-4 over MOZART2 are described below. The chemical mechanism has been significantly expanded to include an improved representation of non-methane hydrocarbons and the online calculation of aerosols. An online photolysis scheme takes into account the impact of aerosols and clouds on the photolysis rates. Dry deposition velocities can be determined online in the model and an improved scheme for the determination of albedo has been implemented.

\subsection{Chemical mechanism}

The standard MOZART-4 mechanism includes 85 gas-phase species, 12 bulk aerosol compounds, 39 photolysis and 157 gas-phase reactions. The chemical mechanism includes an updated isoprene oxidation scheme and a better treatment of volatile organic compounds, with three lumped species to represent alkanes and alkenes with four or more carbon atoms and aromatic compounds (called BIGALK, BIGENE and TOLUENE). Oxidation products of these lumped species have also been added. MOZART-2 had a single lumped species ("C4H10") representing all hydrocarbons $\mathrm{C}_{4}$ and larger. The full list of species and gas-phase, aerosol and photolytic reactions are given in Tables 2-6. Reaction rates are based on JPL 2002 (Sander et al., 2003). Discussion of the isoprene oxidation scheme is given in Pfister et al. (2008a).

Table 7 gives an approximate mapping of the longer-lived VOCs in MOZART-4 to three other mechanisms commonly used in regional models, SAPRC-99, RADM, CBMZ (e.g., Tang et al., 2007; Grell et al., 2005; Fast et al., 2006). One application of this mapping is the matching of MOZART-4 results to regional model boundary conditions.

The MOZART models make use of a chemical preprocessor, making it relatively easy to modify the chemical mechanism. This facilitates updating reaction rates, adding additional species, or running with simplified mechanisms or a few artificial tracers. MOZART-4 also has the capability of reading offline constituents, such as $\mathrm{OH}$, and prescribed chemical production and loss rates, allowing the simulation of species with relatively simple chemistry (e.g., $\mathrm{CO}$ or $\mathrm{CH}_{4}$ ) without running the full chemistry. 
Table 2. Chemical species in MOZART-4.

\begin{tabular}{|c|c|c|c|}
\hline & Symbolic Name & Atomic Composition & Comments \\
\hline $\mathrm{O}, \mathrm{H}, \mathrm{N}$ species & $\begin{array}{l}\mathrm{O} \\
\mathrm{O} 1 \mathrm{D} \\
\mathrm{O} 3 \\
\mathrm{~N} 2 \mathrm{O} \\
\mathrm{NO} \\
\mathrm{NO} 2 \\
\mathrm{NO} 3 \\
\mathrm{HNO} 3 \\
\mathrm{HO} 2 \mathrm{NO} 2 \\
\mathrm{~N} 2 \mathrm{O} 5 \\
\mathrm{H} 2 \\
\mathrm{OH} \\
\mathrm{HO} 2 \\
\mathrm{H} 2 \mathrm{O} 2\end{array}$ & $\begin{array}{l}\mathrm{O}\left({ }^{3} \mathrm{P}\right) \\
\mathrm{O}\left({ }^{1} \mathrm{D}\right) \\
\mathrm{O}_{3} \\
\mathrm{~N}_{2} \mathrm{O} \\
\mathrm{NO} \\
\mathrm{NO}_{2} \\
\mathrm{NO}_{3} \\
\mathrm{HNO}_{3} \\
\mathrm{HNO}_{4} \\
\mathrm{~N}_{2} \mathrm{O}_{5} \\
\mathrm{H}_{2} \\
\mathrm{OH} \\
\mathrm{HO}_{2} \\
\mathrm{H}_{2} \mathrm{O}_{2}\end{array}$ & $\begin{array}{l}\text { ground state atomic oxygen } \\
\text { excited state atomic oxygen } \\
\text { ozone } \\
\text { nitrous oxide } \\
\text { nitric oxide } \\
\text { nitrogen dioxide } \\
\text { nitrate radical } \\
\text { nitric acid } \\
\text { pernitric acid } \\
\text { dinitrogen pentoxide } \\
\text { molecular hydrogen } \\
\text { hydroxyl radical } \\
\text { hydroperoxyl radical } \\
\text { hydrogen peroxide }\end{array}$ \\
\hline $\mathrm{C}_{1}$ species & $\begin{array}{l}\mathrm{CH} 4 \\
\mathrm{CO} \\
\mathrm{CH} 3 \mathrm{O} 2 \\
\mathrm{CH} 3 \mathrm{OOH} \\
\mathrm{CH} 2 \mathrm{O} \\
\mathrm{CH} 3 \mathrm{OH}\end{array}$ & $\begin{array}{l}\mathrm{CH}_{4} \\
\mathrm{CO} \\
\mathrm{CH}_{3} \mathrm{O}_{2} \\
\mathrm{CH}_{3} \mathrm{OOH} \\
\mathrm{CH}_{2} \mathrm{O} \\
\mathrm{CH}_{3} \mathrm{OH}\end{array}$ & $\begin{array}{l}\text { methane } \\
\text { carbon monoxide } \\
\text { methylperoxy radical } \\
\text { methyl hydroperoxide } \\
\text { formaldehyde } \\
\text { methanol }\end{array}$ \\
\hline $\mathrm{C}_{2}$ species & $\begin{array}{l}\mathrm{C} 2 \mathrm{H} 4 \\
\mathrm{C} 2 \mathrm{H} 6 \\
\mathrm{CH} 3 \mathrm{CHO} \\
\mathrm{C} 2 \mathrm{H} 5 \mathrm{OH} \\
\text { EO } \\
\mathrm{EO} 2 \\
\mathrm{CH} 3 \mathrm{COOH} \\
\text { GLYOXAL } \\
\text { GLYALD } \\
\text { C2H5O2 } \\
\text { C2H5OOH } \\
\text { CH3CO3 } \\
\text { CH3COOOH } \\
\text { PAN }\end{array}$ & $\begin{array}{l}\mathrm{C}_{2} \mathrm{H}_{2} \\
\mathrm{C}_{2} \mathrm{H}_{6} \\
\mathrm{CH}_{3} \mathrm{CHO} \\
\mathrm{C}_{2} \mathrm{H}_{5} \mathrm{OH} \\
\mathrm{HOCH}_{2} \mathrm{CH}_{2} \mathrm{O} \\
\mathrm{HOCH}_{2} \mathrm{CH}_{2} \mathrm{O}_{2} \\
\mathrm{CH}_{3} \mathrm{COOH} \\
\mathrm{HCOCHO} \\
\mathrm{HOCH}_{2} \mathrm{CHO} \\
\mathrm{C}_{2} \mathrm{H}_{5} \mathrm{O}_{2} \\
\mathrm{C}_{2} \mathrm{H}_{5} \mathrm{OOH} \\
\mathrm{CH}_{3} \mathrm{CO}_{3} \\
\left.\mathrm{CH}_{3} \mathrm{C}_{3} \mathrm{O}\right) \mathrm{OOH} \\
\mathrm{CH}_{3} \mathrm{CO}_{3} \mathrm{NO}_{2}\end{array}$ & $\begin{array}{l}\text { acetic acid } \\
\text { glyoxal } \\
\text { glycolaldehyde } \\
\text { ethylperoxy radical } \\
\text { ethyl hydroperoxide } \\
\text { acetylperoxy radical } \\
\text { peracetic acid } \\
\text { peroxy acetyl nitrate }\end{array}$ \\
\hline $\mathrm{C}_{3}$ species & $\begin{array}{l}\mathrm{C} 3 \mathrm{H} 6 \\
\mathrm{C} 3 \mathrm{H} 8 \\
\mathrm{C} 3 \mathrm{H} 7 \mathrm{O} 2 \\
\mathrm{C} 3 \mathrm{H} 7 \mathrm{OH} \\
\mathrm{PO} 2 \\
\mathrm{POOH} \\
\mathrm{CH} 3 \mathrm{COCH} 3 \\
\mathrm{HYAC} \\
\mathrm{CH} 3 \mathrm{COCHO} \\
\text { RO2 } \\
\text { ROOH } \\
\text { ONIT }\end{array}$ & $\begin{array}{l}\mathrm{C}_{3} \mathrm{H}_{6} \\
\mathrm{C}_{3} \mathrm{H}_{8} \\
\mathrm{C}_{3} \mathrm{H}_{7} \mathrm{O}_{2} \\
\mathrm{C}_{3} \mathrm{H}_{7} \mathrm{OOH} \\
\text { e.g., } \mathrm{CH}_{3} \mathrm{CH}(\mathrm{OO}) \mathrm{CH}_{2} \mathrm{OH} \\
\text { e.g., } \mathrm{CH}_{3} \mathrm{CH}(\mathrm{OOH}) \mathrm{CH}_{2} \mathrm{OH} \\
\mathrm{CH}_{3} \mathrm{COCH}_{3} \\
\mathrm{CH}_{3} \mathrm{COCH}_{2} \mathrm{OH} \\
\mathrm{CH}_{3} \mathrm{COCHO} \\
\mathrm{CH}_{3} \mathrm{COCH}_{2} \mathrm{O}_{2} \\
\mathrm{CH}_{3} \mathrm{COCH}_{2} \mathrm{OOH}_{2} \\
\mathrm{CH}_{3} \mathrm{COCH}_{2} \mathrm{ONO}_{2}\end{array}$ & $\begin{array}{l}\text { acetone } \\
\text { hydroxyacetone } \\
\text { methylglyoxal }\end{array}$ \\
\hline $\mathrm{C}_{4}$ species & $\begin{array}{l}\text { BIGENE } \\
\text { ENEO2 } \\
\text { MEK } \\
\text { MEKO2 } \\
\text { MEKOOH } \\
\text { MVK } \\
\text { MACR } \\
\text { MPAN } \\
\text { MACRO2 } \\
\text { MACROOH } \\
\text { MCO3 }\end{array}$ & 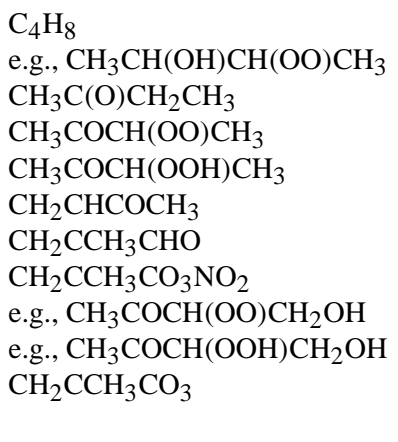 & $\begin{array}{l}\text { lumped alkenes } \mathrm{C}>3 \\
\text { methyl ethyl ketone } \\
\text { methyl vinyl ketone } \\
\text { methacrolein } \\
\text { methacryloyl peroxynitrate } \\
\text { peroxy radical from OH addition to MVK, MACR } \\
\text { peroxy radical derived from abstraction reaction } \\
\text { of OH with MACR }\end{array}$ \\
\hline
\end{tabular}


Table 2. Continued.

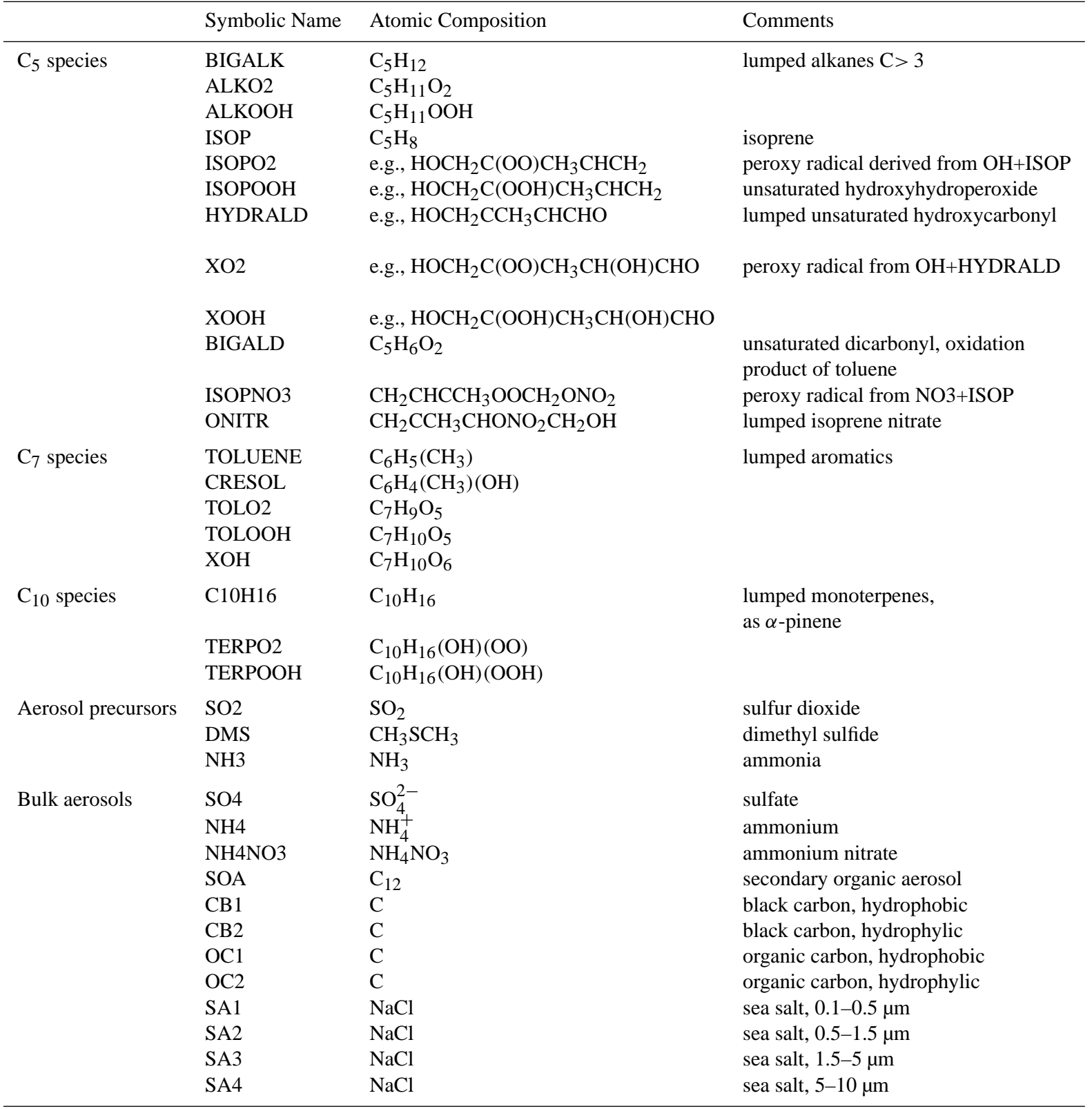

Table 3. Gas-phase reactions. (\{CO2\} indicates $\mathrm{CO} 2$ is not included in the model solution.)

\begin{tabular}{llll}
\hline Reactants & & Products & Rate \\
\hline $\mathrm{O}+\mathrm{O} 2+\mathrm{M}$ & $\rightarrow$ & $\mathrm{O} 3+\mathrm{M}$ & $6 \mathrm{E}-34 \cdot(300 / \mathrm{T})^{2.4}$ \\
$\mathrm{O}+\mathrm{O} 3$ & $\rightarrow$ & $2 \cdot \mathrm{O} 2$ & $8.00 \mathrm{E}-12 \cdot \exp (-2060 / \mathrm{T})$ \\
$\mathrm{O} 1 \mathrm{D}+\mathrm{N} 2$ & $\rightarrow$ & $\mathrm{O}+\mathrm{N} 2$ & $2.10 \mathrm{E}-11 \cdot \exp (115 / \mathrm{T})$ \\
$\mathrm{O} 1 \mathrm{D}+\mathrm{O} 2$ & $\rightarrow$ & $\mathrm{O}+\mathrm{O} 2$ & $3.20 \mathrm{E}-11 \cdot \exp (70 / \mathrm{T})$ \\
$\mathrm{O} 1 \mathrm{D}+\mathrm{H} 2 \mathrm{O}$ & $\rightarrow$ & $2 \cdot \mathrm{OH}$ & $2.20 \mathrm{E}-10$ \\
$\mathrm{H} 2+\mathrm{O} 1 \mathrm{D}$ & $\rightarrow$ & $\mathrm{HO} 2+\mathrm{OH}$ & $1.10 \mathrm{E}-10$ \\
$\mathrm{H} 2+\mathrm{OH}$ & $\rightarrow$ & $\mathrm{H} 2 \mathrm{O}+\mathrm{HO} 2$ & $5.50 \mathrm{E}-12 \cdot \exp (-2000 / \mathrm{T})$ \\
$\mathrm{O}+\mathrm{OH}$ & $\rightarrow$ & $\mathrm{HO} 2+\mathrm{O} 2$ & $2.20 \mathrm{E}-11 \cdot \exp (120 / \mathrm{T})$ \\
$\mathrm{HO} 2+\mathrm{O}$ & $\rightarrow$ & $\mathrm{OH}+\mathrm{O} 2$ & $3.00 \mathrm{E}-11 \cdot \exp (200 / \mathrm{T})$ \\
$\mathrm{OH}+\mathrm{O} 3$ & $\rightarrow$ & $\mathrm{HO} 2+\mathrm{O} 2$ & $1.70 \mathrm{E}-12 \cdot \exp (-940 / \mathrm{T})$ \\
\hline
\end{tabular}


Table 3. Continued.

\begin{tabular}{|c|c|c|c|}
\hline Reactants & & Products & Rate \\
\hline $\mathrm{HO} 2+\mathrm{O} 3$ & $\rightarrow$ & $\mathrm{OH}+2 \cdot \mathrm{O} 2$ & $1.00 \mathrm{E}-14 \cdot \exp (-490 / \mathrm{T})$ \\
\hline $\mathrm{HO} 2+\mathrm{HO} 2$ & $\rightarrow$ & $\mathrm{H} 2 \mathrm{O} 2+\mathrm{O} 2$ & $\begin{array}{l}(2.3 \mathrm{E}-13 \cdot \exp (600 / \mathrm{T})+1.7 \mathrm{E}-33 \cdot[\mathrm{M}] \cdot \exp (1000 / \mathrm{T})) \\
(1+1.4 \mathrm{E}-21 \cdot[\mathrm{H} 2 \mathrm{O}] \cdot \exp (2200 / \mathrm{T}))\end{array}$ \\
\hline $\mathrm{H} 2 \mathrm{O} 2+\mathrm{OH}$ & $\rightarrow$ & $\mathrm{H} 2 \mathrm{O}+\mathrm{HO} 2$ & $2.90 \mathrm{E}-12 \cdot \exp (-160 / \mathrm{T})$ \\
\hline $\mathrm{OH}+\mathrm{HO} 2$ & $\rightarrow$ & $\mathrm{H} 2 \mathrm{O}+\mathrm{O} 2$ & $4.80 \mathrm{E}-11 \cdot \exp (250 / \mathrm{T})$ \\
\hline $\mathrm{OH}+\mathrm{OH}$ & $\rightarrow$ & $\mathrm{H} 2 \mathrm{O}+\mathrm{O}$ & $4.20 \mathrm{E}-12 \cdot \exp (-240 / \mathrm{T})$ \\
\hline $\mathrm{OH}+\mathrm{OH}+\mathrm{M}$ & $\rightarrow$ & $\mathrm{H} 2 \mathrm{O} 2+\mathrm{M}$ & $\mathrm{ko}=6.90 \mathrm{E}-31 \cdot(300 / \mathrm{T})^{1.00} ; \mathrm{ki}=2.60 \mathrm{E}-11 ; \mathrm{f}=0.60$ \\
\hline $\mathrm{N} 2 \mathrm{O}+\mathrm{O} 1 \mathrm{D}$ & $\rightarrow$ & $\mathrm{N} 2+\mathrm{O} 2$ & $4.90 \mathrm{E}-11$ \\
\hline $\mathrm{N} 2 \mathrm{O}+\mathrm{O} 1 \mathrm{D}$ & $\rightarrow$ & $2 \cdot \mathrm{NO}$ & $6.70 \mathrm{E}-11$ \\
\hline $\mathrm{NO}+\mathrm{HO} 2$ & $\rightarrow$ & $\mathrm{NO} 2+\mathrm{OH}$ & $3.50 \mathrm{E}-12 \cdot \exp (250 / \mathrm{T})$ \\
\hline $\mathrm{NO}+\mathrm{O} 3$ & $\rightarrow$ & $\mathrm{NO} 2+\mathrm{O} 2$ & $3.00 \mathrm{E}-12 \cdot \exp (-1500 / \mathrm{T})$ \\
\hline $\mathrm{NO} 2+\mathrm{O}$ & $\rightarrow$ & $\mathrm{NO}+\mathrm{O} 2$ & $5.60 \mathrm{E}-12 \cdot \exp (180 / \mathrm{T})$ \\
\hline $\mathrm{NO} 2+\mathrm{O} 3$ & $\rightarrow$ & $\mathrm{NO} 3+\mathrm{O} 2$ & $1.20 \mathrm{E}-13 \cdot \exp (-2450 / \mathrm{T})$ \\
\hline $\mathrm{NO} 3+\mathrm{HO} 2$ & $\rightarrow$ & $\mathrm{OH}+\mathrm{NO} 2$ & $2.30 \mathrm{E}-12 \cdot \exp (170 / \mathrm{T})$ \\
\hline $\mathrm{NO} 2+\mathrm{NO} 3+\mathrm{M}$ & $\rightarrow$ & $\mathrm{N} 2 \mathrm{O} 5+\mathrm{M}$ & $\mathrm{ko}=2.00 \mathrm{E}-30 \cdot(300 / \mathrm{T})^{4.40} ; \mathrm{ki}=1.40 \mathrm{E}-12 \cdot(300 / \mathrm{T})^{0.70} ; \mathrm{f}=0.60$ \\
\hline $\mathrm{N} 2 \mathrm{O} 5+\mathrm{M}$ & $\rightarrow$ & $\mathrm{NO} 2+\mathrm{NO} 3+\mathrm{M}$ & $\mathrm{k}(\mathrm{NO} 2+\mathrm{NO} 3+\mathrm{M}) \cdot 3.333 \mathrm{E} 26 \cdot \exp (-10990 / \mathrm{T})$ \\
\hline $\mathrm{NO} 2+\mathrm{OH}+\mathrm{M}$ & $\rightarrow$ & $\mathrm{HNO} 3+\mathrm{M}$ & $\mathrm{ko}=2.00 \mathrm{E}-30 \cdot(300 / \mathrm{T})^{3.00} ; \mathrm{ki}=2.50 \mathrm{E}-1 ; \mathrm{f}=0.60$ \\
\hline $\mathrm{HNO} 3+\mathrm{OH}$ & $\rightarrow$ & $\mathrm{NO} 3+\mathrm{H} 2 \mathrm{O}$ & $\begin{array}{l}\mathrm{k} 0+\mathrm{k} 3[\mathrm{M}] /(1+\mathrm{k} 3[\mathrm{M}] / \mathrm{k} 2) \\
\mathrm{k} 0=2.4 \mathrm{E}-14 \cdot \exp (460 / \mathrm{T}) \\
\mathrm{k} 2=2.7 \mathrm{E}-17 \cdot \exp (2199 / \mathrm{T}) \\
\mathrm{k} 3=6.5 \mathrm{E}-34 \cdot \exp (1335 / \mathrm{T})\end{array}$ \\
\hline $\mathrm{NO} 3+\mathrm{NO}$ & $\rightarrow$ & $2 \cdot \mathrm{NO} 2$ & $1.50 \mathrm{E}-11 \cdot \exp (170 / \mathrm{T})$ \\
\hline $\mathrm{NO} 2+\mathrm{HO} 2+\mathrm{M}$ & $\rightarrow$ & $\mathrm{HO} 2 \mathrm{NO} 2+\mathrm{M}$ & $\mathrm{ko}=1.80 \mathrm{E}-31 \cdot(300 / \mathrm{T})^{3.20} ; \mathrm{ki}=4.70 \mathrm{E}-12 \cdot(300 / \mathrm{T})^{1.40} ; \mathrm{f}=0.60$ \\
\hline $\mathrm{HO} 2 \mathrm{NO} 2+\mathrm{OH}$ & $\rightarrow$ & $\mathrm{H} 2 \mathrm{O}+\mathrm{NO} 2+\mathrm{O} 2$ & $1.30 \mathrm{E}-12 \cdot \exp (380 / \mathrm{T})$ \\
\hline $\mathrm{HO} 2 \mathrm{NO} 2+\mathrm{M}$ & $\rightarrow$ & $\mathrm{HO} 2+\mathrm{NO} 2+\mathrm{M}$ & $\mathrm{k}(\mathrm{NO} 2+\mathrm{HO} 2+\mathrm{M}) \cdot \exp (-10900 / \mathrm{T}) / 2.1 \mathrm{E}-27$ \\
\hline $\mathrm{CH} 4+\mathrm{OH}$ & $\rightarrow$ & $\mathrm{CH} 3 \mathrm{O} 2+\mathrm{H} 2 \mathrm{O}$ & $2.45 \mathrm{E}-12 \cdot \exp (-1775 / \mathrm{T})$ \\
\hline $\mathrm{CH} 4+\mathrm{O} 1 \mathrm{D}$ & $\rightarrow$ & $\begin{array}{l}.75 \cdot \mathrm{CH} 3 \mathrm{O} 2+.75 \cdot \mathrm{OH}+.25 \cdot \mathrm{CH} 2 \mathrm{O} \\
+.4 \cdot \mathrm{HO} 2+.05 \cdot \mathrm{H} 2\end{array}$ & $1.50 \mathrm{E}-10$ \\
\hline $\mathrm{CH} 3 \mathrm{O} 2+\mathrm{NO}$ & $\rightarrow$ & $\mathrm{CH} 2 \mathrm{O}+\mathrm{NO} 2+\mathrm{HO} 2$ & $2.80 \mathrm{E}-12 \cdot \exp (300 / \mathrm{T})$ \\
\hline $\mathrm{CH} 3 \mathrm{O} 2+\mathrm{CH} 3 \mathrm{O} 2$ & $\rightarrow$ & $2 \cdot \mathrm{CH} 2 \mathrm{O}+2 \cdot \mathrm{HO} 2$ & $5.00 \mathrm{E}-13 \cdot \exp (-424 / \mathrm{T})$ \\
\hline $\mathrm{CH} 3 \mathrm{O} 2+\mathrm{CH} 3 \mathrm{O} 2$ & $\rightarrow$ & $\mathrm{CH} 2 \mathrm{O}+\mathrm{CH} 3 \mathrm{OH}$ & $1.90 \mathrm{E}-14 \cdot \exp (706 / \mathrm{T})$ \\
\hline $\mathrm{CH} 3 \mathrm{O} 2+\mathrm{HO} 2$ & $\rightarrow$ & $\mathrm{CH} 3 \mathrm{OOH}+\mathrm{O} 2$ & $4.10 \mathrm{E}-13 \cdot \exp (750 / \mathrm{T})$ \\
\hline $\mathrm{CH} 3 \mathrm{OOH}+\mathrm{OH}$ & $\rightarrow$ & $.7 \cdot \mathrm{CH} 3 \mathrm{O} 2+.3 \cdot \mathrm{OH}+.3 \cdot \mathrm{CH} 2 \mathrm{O}+\mathrm{H} 2 \mathrm{O}$ & $3.80 \mathrm{E}-12 \cdot \exp (200 / \mathrm{T})$ \\
\hline $\mathrm{CH} 2 \mathrm{O}+\mathrm{NO} 3$ & $\rightarrow$ & $\mathrm{CO}+\mathrm{HO} 2+\mathrm{HNO} 3$ & $6.00 \mathrm{E}-13 \cdot \exp (-2058 / \mathrm{T})$ \\
\hline $\mathrm{CH} 2 \mathrm{O}+\mathrm{OH}$ & $\rightarrow$ & $\mathrm{CO}+\mathrm{H} 2 \mathrm{O}+\mathrm{HO} 2$ & $9.00 \mathrm{E}-12$ \\
\hline $\mathrm{CO}+\mathrm{OH}$ & $\rightarrow$ & $\{\mathrm{CO} 2\}+\mathrm{HO} 2$ & $1.5 \mathrm{E}-13 \cdot(1+6 \mathrm{E}-7 \cdot \mathrm{P})$ \\
\hline $\mathrm{CH} 3 \mathrm{OH}+\mathrm{OH}$ & $\rightarrow$ & $\mathrm{HO} 2+\mathrm{CH} 2 \mathrm{O}$ & $7.30 \mathrm{E}-12 \cdot \exp (-620 / \mathrm{T})$ \\
\hline $\mathrm{C} 2 \mathrm{H} 4+\mathrm{OH}+\mathrm{M}$ & $\rightarrow$ & $.75 \cdot \mathrm{EO} 2+.5 \cdot \mathrm{CH} 2 \mathrm{O}+.25 \cdot \mathrm{HO} 2+\mathrm{M}$ & $\begin{array}{l}\mathrm{ko}=1.00 \mathrm{E}-28 \cdot(300 / \mathrm{T})^{0.80} \\
\mathrm{ki}=8.80 \mathrm{E}-12 ; \mathrm{f}=0.60\end{array}$ \\
\hline $\mathrm{C} 2 \mathrm{H} 4+\mathrm{O} 3$ & $\rightarrow$ & $\begin{array}{l}\mathrm{CH} 2 \mathrm{O}+.12 \cdot \mathrm{HO} 2+.5 \cdot \mathrm{CO} \\
+.12 \cdot \mathrm{OH}+.25 \cdot \mathrm{CH} 3 \mathrm{COOH}\end{array}$ & $1.20 \mathrm{E}-14 \cdot \exp (-2630 / \mathrm{T})$ \\
\hline $\mathrm{EO} 2+\mathrm{NO}$ & $\rightarrow$ & $\mathrm{EO}+\mathrm{NO} 2$ & $4.20 \mathrm{E}-12 \cdot \exp (180 / \mathrm{T})$ \\
\hline $\mathrm{EO}+\mathrm{O} 2$ & $\rightarrow$ & GLYALD + HO2 & $1.00 \mathrm{E}-14$ \\
\hline EO & $\rightarrow$ & $2 \cdot \mathrm{CH} 2 \mathrm{O}+\mathrm{HO} 2$ & $1.60 \mathrm{E}+11 \cdot \exp (-4150 / \mathrm{T})$ \\
\hline $\mathrm{C} 2 \mathrm{H} 6+\mathrm{OH}$ & $\rightarrow$ & $\mathrm{C} 2 \mathrm{H} 5 \mathrm{O} 2+\mathrm{H} 2 \mathrm{O}$ & $8.70 \mathrm{E}-12 \cdot \exp (-1070 / \mathrm{T})$ \\
\hline $\mathrm{C} 2 \mathrm{H} 5 \mathrm{O} 2+\mathrm{NO}$ & $\rightarrow$ & $\mathrm{CH} 3 \mathrm{CHO}+\mathrm{HO} 2+\mathrm{NO} 2$ & $2.60 \mathrm{E}-12 \cdot \exp (365 / \mathrm{T})$ \\
\hline $\mathrm{C} 2 \mathrm{H} 5 \mathrm{O} 2+\mathrm{HO} 2$ & $\rightarrow$ & $\mathrm{C} 2 \mathrm{H} 5 \mathrm{OOH}+\mathrm{O} 2$ & $7.50 \mathrm{E}-13 \cdot \exp (700 / \mathrm{T})$ \\
\hline $\mathrm{C} 2 \mathrm{H} 5 \mathrm{O} 2+\mathrm{CH} 3 \mathrm{O} 2$ & $\rightarrow$ & $\begin{array}{l}.7 \cdot \mathrm{CH} 2 \mathrm{O}+.8 \cdot \mathrm{CH} 3 \mathrm{CHO}+\mathrm{HO} 2 \\
+.3 \cdot \mathrm{CH} 3 \mathrm{OH}+.2 \cdot \mathrm{C} 2 \mathrm{H} 5 \mathrm{OH}\end{array}$ & $2.00 \mathrm{E}-13$ \\
\hline $\mathrm{C} 2 \mathrm{H} 5 \mathrm{O} 2+\mathrm{C} 2 \mathrm{H} 5 \mathrm{O} 2$ & $\rightarrow$ & $1.6 \cdot \mathrm{CH} 3 \mathrm{CHO}+1.2 \cdot \mathrm{HO} 2+.4 \cdot \mathrm{C} 2 \mathrm{H} 5 \mathrm{OH}$ & $6.80 \mathrm{E}-14$ \\
\hline $\mathrm{C} 2 \mathrm{H} 5 \mathrm{OOH}+\mathrm{OH}$ & $\rightarrow$ & $.5 \cdot \mathrm{C} 2 \mathrm{H} 5 \mathrm{O} 2+.5 \cdot \mathrm{CH} 3 \mathrm{CHO}+.5 \cdot \mathrm{OH}$ & $3.80 \mathrm{E}-12 \cdot \exp (200 / \mathrm{T})$ \\
\hline $\mathrm{CH} 3 \mathrm{CHO}+\mathrm{OH}$ & $\rightarrow$ & $\mathrm{CH} 3 \mathrm{CO} 3+\mathrm{H} 2 \mathrm{O}$ & $5.60 \mathrm{E}-12 \cdot \exp (270 / \mathrm{T})$ \\
\hline $\mathrm{CH} 3 \mathrm{CHO}+\mathrm{NO} 3$ & $\rightarrow$ & $\mathrm{CH} 3 \mathrm{CO} 3+\mathrm{HNO} 3$ & $1.40 \mathrm{E}-12 \cdot \exp (-1900 / \mathrm{T})$ \\
\hline $\mathrm{CH} 3 \mathrm{CO} 3+\mathrm{NO}$ & $\rightarrow$ & $\mathrm{CH} 3 \mathrm{O} 2+\{\mathrm{CO} 2\}+\mathrm{NO} 2$ & $8.10 \mathrm{E}-12 \cdot \exp (270 / \mathrm{T})$ \\
\hline $\mathrm{CH} 3 \mathrm{CO} 3+\mathrm{NO} 2+\mathrm{M}$ & $\rightarrow$ & $\mathrm{PAN}+\mathrm{M}$ & $\begin{array}{l}\mathrm{ko}=8.50 \mathrm{E}-29 \cdot(300 / \mathrm{T})^{6.50} \\
\mathrm{ki}=1.10 \mathrm{E}-11 \cdot(300 / \mathrm{T}) ; \mathrm{f}=0.60\end{array}$ \\
\hline $\mathrm{CH} 3 \mathrm{CO} 3+\mathrm{HO} 2$ & $\rightarrow$ & $.75 \cdot \mathrm{CH} 3 \mathrm{COOOH}+.25 \cdot \mathrm{CH} 3 \mathrm{COOH}+.25 \cdot \mathrm{O} 3$ & $4.30 \mathrm{E}-13 \cdot \exp (1040 / \mathrm{T})$ \\
\hline $\mathrm{CH} 3 \mathrm{CO} 3+\mathrm{CH} 3 \mathrm{O} 2$ & $\rightarrow$ & $\begin{array}{l}.9 \cdot \mathrm{CH} 3 \mathrm{O} 2+\mathrm{CH} 2 \mathrm{O}+.9 \cdot \mathrm{HO} 2 \\
+.1 \cdot \mathrm{CH} 3 \mathrm{COOH}+.9 \cdot\{\mathrm{CO} 2\}\end{array}$ & $2.00 \mathrm{E}-12 \cdot \exp (500 / \mathrm{T})$ \\
\hline $\mathrm{CH} 3 \mathrm{COOOH}+\mathrm{OH}$ & $\rightarrow$ & $.5 \cdot \mathrm{CH} 3 \mathrm{CO} 3+.5 \cdot \mathrm{CH} 2 \mathrm{O}+.5 \cdot\{\mathrm{CO} 2\}+\mathrm{H} 2 \mathrm{O}$ & $1.00 \mathrm{E}-12$ \\
\hline $\mathrm{PAN}+\mathrm{OH}$ & $\rightarrow$ & $\mathrm{CH} 2 \mathrm{O}+\mathrm{NO} 3+\{\mathrm{CO} 2\}$ & $4.00 \mathrm{E}-14$ \\
\hline
\end{tabular}


Table 3. Continued.

\begin{tabular}{|c|c|c|c|}
\hline Reactants & & Products & Rate \\
\hline $\mathrm{PAN}+\mathrm{M}$ & $\rightarrow$ & $\mathrm{CH} 3 \mathrm{CO} 3+\mathrm{NO} 2+\mathrm{M}$ & $\begin{array}{l}\mathrm{k}(\mathrm{CH} 3 \mathrm{CO} 3+\mathrm{NO} 2+\mathrm{M}) \cdot 1.111 \mathrm{E} 28 \\
\cdot \exp (-14000 / \mathrm{T})\end{array}$ \\
\hline $\mathrm{CH} 3 \mathrm{CO} 3+\mathrm{CH} 3 \mathrm{CO} 3$ & $\rightarrow$ & $2 \cdot \mathrm{CH} 3 \mathrm{O} 2+2 \cdot\{\mathrm{CO} 2\}$ & $2.50 \mathrm{E}-12 \cdot \exp (500 / \mathrm{T})$ \\
\hline GLYALD + OH & $\rightarrow$ & $\mathrm{HO} 2+.2 \cdot \mathrm{GLYOXAL}+.8 \cdot \mathrm{CH} 2 \mathrm{O}+.8 \cdot\{\mathrm{CO} 2\}$ & $1.00 \mathrm{E}-11$ \\
\hline GLYOXAL + OH & $\rightarrow$ & $\mathrm{HO} 2+\mathrm{CO}+\{\mathrm{CO} 2\}$ & $1.10 \mathrm{E}-11$ \\
\hline $\mathrm{CH} 3 \mathrm{COOH}+\mathrm{OH}$ & $\rightarrow$ & $\mathrm{CH} 3 \mathrm{O} 2+\{\mathrm{CO} 2\}+\mathrm{H} 2 \mathrm{O}$ & $7.00 \mathrm{E}-13$ \\
\hline $\mathrm{C} 2 \mathrm{H} 5 \mathrm{OH}+\mathrm{OH}$ & $\rightarrow$ & $\mathrm{HO} 2+\mathrm{CH} 3 \mathrm{CHO}$ & $6.90 \mathrm{E}-12 \cdot \exp (-230 / \mathrm{T})$ \\
\hline $\mathrm{C} 3 \mathrm{H} 6+\mathrm{OH}+\mathrm{M}$ & $\rightarrow$ & $\mathrm{PO} 2+\mathrm{M}$ & $\begin{array}{l}\mathrm{ko}=8.00 \mathrm{E}-27 \cdot(300 / \mathrm{T})^{3.50} \\
\mathrm{ki}=3.00 \mathrm{E}-11 ; \mathrm{f}=0.50\end{array}$ \\
\hline $\mathrm{C} 3 \mathrm{H} 6+\mathrm{O} 3$ & $\rightarrow$ & $\begin{array}{l}.54 \cdot \mathrm{CH} 2 \mathrm{O}+.19 \cdot \mathrm{HO} 2+.33 \cdot \mathrm{OH} \\
+.5 \cdot \mathrm{CH} 3 \mathrm{CHO}+.56 \cdot \mathrm{CO}+.31 \cdot \mathrm{CH} 3 \mathrm{O} 2 \\
+.25 \cdot \mathrm{CH} 3 \mathrm{COOH}+.08 \cdot \mathrm{CH} 4\end{array}$ & $6.50 \mathrm{E}-15 \cdot \exp (-1900 / \mathrm{T})$ \\
\hline $\mathrm{C} 3 \mathrm{H} 6+\mathrm{NO} 3$ & $\rightarrow$ & ONIT & $4.60 \mathrm{E}-13 \cdot \exp (-1156 / \mathrm{T})$ \\
\hline $\mathrm{PO} 2+\mathrm{NO}$ & $\rightarrow$ & $\mathrm{CH} 3 \mathrm{CHO}+\mathrm{CH} 2 \mathrm{O}+\mathrm{HO} 2+\mathrm{NO} 2$ & $4.20 \mathrm{E}-12 \cdot \exp (180 / \mathrm{T})$ \\
\hline $\mathrm{PO} 2+\mathrm{HO} 2$ & $\rightarrow$ & $\mathrm{POOH}+\mathrm{O} 2$ & $7.50 \mathrm{E}-13 \cdot \exp (700 / \mathrm{T})$ \\
\hline $\mathrm{POOH}+\mathrm{OH}$ & $\rightarrow$ & $.5 \cdot \mathrm{PO} 2+.5 \cdot \mathrm{OH}+.5 \cdot \mathrm{HYAC}+\mathrm{H} 2 \mathrm{O}$ & $3.80 \mathrm{E}-12 \cdot \exp (200 / \mathrm{T})$ \\
\hline $\mathrm{C} 3 \mathrm{H} 8+\mathrm{OH}$ & $\rightarrow$ & $\mathrm{C} 3 \mathrm{H} 7 \mathrm{O} 2+\mathrm{H} 2 \mathrm{O}$ & $1.00 \mathrm{E}-11 \cdot \exp (-660 / \mathrm{T})$ \\
\hline $\mathrm{C} 3 \mathrm{H} 7 \mathrm{O} 2+\mathrm{NO}$ & $\rightarrow$ & $.82 \cdot \mathrm{CH} 3 \mathrm{COCH} 3+\mathrm{NO} 2+.27 \cdot \mathrm{CH} 3 \mathrm{CHO}+\mathrm{HO} 2$ & $4.20 \mathrm{E}-12 \cdot \exp (180 / \mathrm{T})$ \\
\hline $\mathrm{C} 3 \mathrm{H} 7 \mathrm{O} 2+\mathrm{HO} 2$ & $\rightarrow$ & $\mathrm{C} 3 \mathrm{H} 7 \mathrm{OOH}+\mathrm{O} 2$ & $7.50 \mathrm{E}-13 \cdot \exp (700 / \mathrm{T})$ \\
\hline $\mathrm{C} 3 \mathrm{H} 7 \mathrm{O} 2+\mathrm{CH} 3 \mathrm{O} 2$ & $\rightarrow$ & $\mathrm{CH} 2 \mathrm{O}+\mathrm{HO} 2+.82 \cdot \mathrm{CH} 3 \mathrm{COCH} 3$ & $3.75 \mathrm{E}-13 \cdot \exp (-40 / \mathrm{T})$ \\
\hline $\mathrm{C} 3 \mathrm{H} 7 \mathrm{OOH}+\mathrm{OH}$ & $\rightarrow$ & $\mathrm{H} 2 \mathrm{O}+\mathrm{C} 3 \mathrm{H} 7 \mathrm{O} 2$ & $3.80 \mathrm{E}-12 \cdot \exp (200 / \mathrm{T})$ \\
\hline $\mathrm{CH} 3 \mathrm{COCH} 3+\mathrm{OH}$ & $\rightarrow$ & $\mathrm{RO} 2+\mathrm{H} 2 \mathrm{O}$ & $3.82 \mathrm{E}-11 \cdot \exp (-2000 / \mathrm{T})+1.33 \mathrm{E}-13$ \\
\hline $\mathrm{RO} 2+\mathrm{NO}$ & $\rightarrow$ & $\mathrm{CH} 3 \mathrm{CO} 3+\mathrm{CH} 2 \mathrm{O}+\mathrm{NO} 2$ & $2.90 \mathrm{E}-12 \cdot \exp (300 / \mathrm{T})$ \\
\hline $\mathrm{RO} 2+\mathrm{HO} 2$ & $\rightarrow$ & $\mathrm{ROOH}+\mathrm{O} 2$ & $8.60 \mathrm{E}-13 \cdot \exp (700 / \mathrm{T})$ \\
\hline $\mathrm{RO} 2+\mathrm{CH} 3 \mathrm{O} 2$ & $\rightarrow$ & $\begin{array}{l}.3 \cdot \mathrm{CH} 3 \mathrm{CO} 3+.2 \cdot \mathrm{HYAC}+.8 \cdot \mathrm{CH} 2 \mathrm{O} \\
+.5 \cdot \mathrm{CH} 3 \mathrm{OH}+.3 \cdot \mathrm{HO} 2+.5 \cdot \mathrm{CH} 3 \mathrm{COCHO}\end{array}$ & $2.00 \mathrm{E}-12 \cdot \exp (500 / \mathrm{T})$ \\
\hline $\mathrm{ROOH}+\mathrm{OH}$ & $\rightarrow$ & $\mathrm{RO} 2+\mathrm{H} 2 \mathrm{O}$ & $3.80 \mathrm{E}-12 \cdot \exp (200 / \mathrm{T})$ \\
\hline $\mathrm{ONIT}+\mathrm{OH}$ & $\rightarrow$ & $\mathrm{NO} 2+\mathrm{CH} 3 \mathrm{COCHO}$ & $6.80 \mathrm{E}-13$ \\
\hline $\mathrm{CH} 3 \mathrm{COCHO}+\mathrm{OH}$ & $\rightarrow$ & $\mathrm{CH} 3 \mathrm{CO} 3+\mathrm{CO}+\mathrm{H} 2 \mathrm{O}$ & $8.40 \mathrm{E}-13 \cdot \exp (830 / \mathrm{T})$ \\
\hline $\mathrm{CH} 3 \mathrm{COCHO}+\mathrm{NO} 3$ & $\rightarrow$ & $\mathrm{HNO} 3+\mathrm{CO}+\mathrm{CH} 3 \mathrm{CO} 3$ & $1.40 \mathrm{E}-12 \cdot \exp (-1860 / \mathrm{T})$ \\
\hline $\mathrm{HYAC}+\mathrm{OH}$ & $\rightarrow$ & $\mathrm{CH} 3 \mathrm{COCHO}+\mathrm{HO} 2$ & $3.00 \mathrm{E}-12$ \\
\hline $\mathrm{BIGENE}+\mathrm{OH}$ & $\rightarrow$ & ENEO2 & $5.40 \mathrm{E}-11$ \\
\hline $\mathrm{ENEO} 2+\mathrm{NO}$ & $\rightarrow$ & $\mathrm{CH} 3 \mathrm{CHO}+.5 \cdot \mathrm{CH} 2 \mathrm{O}+.5 \cdot \mathrm{CH} 3 \mathrm{COCH} 3+\mathrm{HO} 2+\mathrm{NO} 2$ & $4.20 \mathrm{E}-12 \cdot \exp (180 / \mathrm{T})$ \\
\hline $\mathrm{MEK}+\mathrm{OH}$ & $\rightarrow$ & $\mathrm{MEKO} 2$ & $2.30 \mathrm{E}-12 \cdot \exp (-170 / \mathrm{T})$ \\
\hline $\mathrm{MEKO} 2+\mathrm{NO}$ & $\rightarrow$ & $\mathrm{CH} 3 \mathrm{CO} 3+\mathrm{CH} 3 \mathrm{CHO}+\mathrm{NO} 2$ & $4.20 \mathrm{E}-12 \cdot \exp (180 / \mathrm{T})$ \\
\hline $\mathrm{MEKO} 2+\mathrm{HO} 2$ & $\rightarrow$ & MEKOOH & $7.50 \mathrm{E}-13 \cdot \exp (700 / \mathrm{T})$ \\
\hline $\mathrm{MEKOOH}+\mathrm{OH}$ & $\rightarrow$ & MEKO2 & $3.80 \mathrm{E}-12 \cdot \exp (200 / \mathrm{T})$ \\
\hline $\mathrm{MPAN}+\mathrm{OH}$ & $\rightarrow$ & $\begin{array}{l}.5 \cdot \mathrm{HYAC}+.5 \cdot \mathrm{NO} 3+.5 \cdot \mathrm{CH} 2 \mathrm{O} \\
+.5 \cdot \mathrm{HO} 2+.5 \cdot\{\mathrm{CO} 2\}\end{array}$ & $\begin{array}{l}\mathrm{ko}=8.00 \mathrm{E}-27 \cdot(300 / \mathrm{T})^{3.50} \\
\mathrm{ki}=3.00 \mathrm{E}-11 ; \mathrm{f}=0.50\end{array}$ \\
\hline $\mathrm{BIGALK}+\mathrm{OH}$ & $\rightarrow$ & $\mathrm{ALKO} 2$ & $3.50 \mathrm{E}-12$ \\
\hline $\mathrm{ALKO} 2+\mathrm{NO}$ & $\rightarrow$ & $\begin{array}{l}.4 \cdot \mathrm{CH} 3 \mathrm{CHO}+.1 \cdot \mathrm{CH} 2 \mathrm{O}+.25 \cdot \mathrm{CH} 3 \mathrm{COCH} 3 \\
+.9 \cdot \mathrm{HO} 2+.75 \cdot \mathrm{MEK}+.9 \cdot \mathrm{NO} 2+.1 \cdot \mathrm{ONIT}\end{array}$ & $4.20 \mathrm{E}-12 \cdot \exp (180 / \mathrm{T})$ \\
\hline $\mathrm{ALKO} 2+\mathrm{HO} 2$ & $\rightarrow$ & ALKOOH & $7.50 \mathrm{E}-13 \cdot \exp (700 / \mathrm{T})$ \\
\hline $\mathrm{ALKOOH}+\mathrm{OH}$ & $\rightarrow$ & ALKO2 & $3.80 \mathrm{E}-12 \cdot \exp (200 / \mathrm{T})$ \\
\hline $\mathrm{ISOP}+\mathrm{OH}$ & $\rightarrow$ & ISOPO 2 & $2.54 \mathrm{E}-11 \cdot \exp (410 / \mathrm{T})$ \\
\hline $\mathrm{ISOP}+\mathrm{O} 3$ & $\rightarrow$ & $\begin{array}{l}.4 \cdot \mathrm{MACR}+.2 \cdot \mathrm{MVK}+.07 \cdot \mathrm{C} 3 \mathrm{H} 6+.27 \cdot \mathrm{OH} \\
+.06 \cdot \mathrm{HO} 2+.6 \cdot \mathrm{CH} 2 \mathrm{O}+.3 \cdot \mathrm{CO} \\
+.1 \cdot \mathrm{O} 3+.2 \cdot \mathrm{MCO} 3+.2 \cdot \mathrm{CH} 3 \mathrm{COOH}\end{array}$ & $1.05 \mathrm{E}-14 \cdot \exp (-2000 / \mathrm{T})$ \\
\hline ISOPO2 + NO & $\rightarrow$ & $\begin{array}{l}.08 \cdot \mathrm{ONITR}+.92 \cdot \mathrm{NO} 2+\mathrm{HO} 2+.55 \cdot \mathrm{CH} 2 \mathrm{O} \\
+.23 \cdot \mathrm{MACR}+.32 \cdot \mathrm{MVK}+.37 \cdot \mathrm{HYDRALD}\end{array}$ & $4.40 \mathrm{E}-12 \cdot \exp (180 / \mathrm{T})$ \\
\hline $\mathrm{ISOPO} 2+\mathrm{NO} 3$ & $\rightarrow$ & $\begin{array}{l}\mathrm{HO} 2+\mathrm{NO} 2+.6 \cdot \mathrm{CH} 2 \mathrm{O}+.25 \cdot \mathrm{MACR} \\
+.35 \cdot \mathrm{MVK}+.4 \cdot \mathrm{HYDRALD}\end{array}$ & $2.40 \mathrm{E}-12$ \\
\hline $\mathrm{ISOPO} 2+\mathrm{HO} 2$ & $\rightarrow$ & ISOPOOH & $8.00 \mathrm{E}-13 \cdot \exp (700 / \mathrm{T})$ \\
\hline ISOPOOH + OH & $\rightarrow$ & $.5 \cdot \mathrm{XO} 2+.5 \cdot \mathrm{ISOPO} 2$ & $1.52 \mathrm{E}-11 \cdot \exp (200 / \mathrm{T})$ \\
\hline $\mathrm{ISOPO} 2+\mathrm{CH} 3 \mathrm{O} 2$ & $\rightarrow$ & $\begin{array}{l}1.2 \cdot \mathrm{CH} 2 \mathrm{O}+.19 \cdot \mathrm{MACR}+.26 \cdot \mathrm{MVK}+.3 \cdot \mathrm{HYDRALD} \\
+.25 \cdot \mathrm{CH} 3 \mathrm{OH}+\mathrm{HO} 2\end{array}$ & $5.00 \mathrm{E}-13 \cdot \exp (400 / \mathrm{T})$ \\
\hline
\end{tabular}


Table 3. Continued.

\begin{tabular}{|c|c|c|c|}
\hline Reactants & & Products & Rate \\
\hline $\mathrm{ISOPO} 2+\mathrm{CH} 3 \mathrm{CO} 3$ & $\rightarrow$ & $\begin{array}{l}.6 \cdot \mathrm{CH} 2 \mathrm{O}+.25 \cdot \mathrm{MACR}+.35 \cdot \mathrm{MVK} \\
+.4 \cdot \mathrm{HYDRALD}+\mathrm{CH} 3 \mathrm{O} 2+\mathrm{HO} 2+\{\mathrm{CO} 2\}\end{array}$ & $1.40 \mathrm{E}-11$ \\
\hline ISOP + NO3 & $\rightarrow$ & ISOPNO3 & $3.03 \mathrm{E}-12 \cdot \exp (-446 / \mathrm{T})$ \\
\hline ISOPNO3 + NO & $\rightarrow$ & $\begin{array}{l}1.206 \cdot \mathrm{NO} 2+.072 \cdot \mathrm{CH} 2 \mathrm{O}+.167 \cdot \mathrm{MACR} \\
+.039 \cdot \mathrm{MVK}+.794 \cdot \mathrm{ONITR}+.794 \cdot \mathrm{HO} 2\end{array}$ & $2.70 \mathrm{E}-12 \cdot \exp (360 / \mathrm{T})$ \\
\hline ISOPNO3 + NO3 & $\rightarrow$ & $\begin{array}{l}1.206 \cdot \mathrm{NO} 2+.072 \cdot \mathrm{CH} 2 \mathrm{O}+.167 \cdot \mathrm{MACR} \\
+.039 \cdot \mathrm{MVK}+.794 \cdot \mathrm{ONITR}+.794 \cdot \mathrm{HO} 2\end{array}$ & $2.40 \mathrm{E}-12$ \\
\hline ISOPNO3 + HO2 & $\rightarrow$ & $\begin{array}{l}.206 \cdot \mathrm{NO} 2+.008 \cdot \mathrm{CH} 2 \mathrm{O}+.167 \cdot \mathrm{MACR} \\
+.039 \cdot \mathrm{MVK}+.794 \cdot \mathrm{ONITR}+.794 \cdot \mathrm{HO} 2\end{array}$ & $8.00 \mathrm{E}-13 \cdot \exp (700 / \mathrm{T})$ \\
\hline $\mathrm{MVK}+\mathrm{OH}$ & $\rightarrow$ & MACRO2 & $4.13 \mathrm{E}-12 \cdot \exp (452 / \mathrm{T})$ \\
\hline $\mathrm{MVK}+\mathrm{O} 3$ & $\rightarrow$ & $\begin{array}{l}.8 \cdot \mathrm{CH} 2 \mathrm{O}+.95 \cdot \mathrm{CH} 3 \mathrm{COCHO}+.08 \cdot \mathrm{OH}+.2 \cdot \mathrm{O} 3 \\
+.06 \cdot \mathrm{HO} 2+.05 \cdot \mathrm{CO}+.04 \cdot \mathrm{CH} 3 \mathrm{CHO}\end{array}$ & $7.52 \mathrm{E}-16 \cdot \exp (-1521 / \mathrm{T})$ \\
\hline $\mathrm{MACR}+\mathrm{OH}$ & $\rightarrow$ & $.5 \cdot \mathrm{MACRO} 2+.5 \cdot \mathrm{H} 2 \mathrm{O}+.5 \cdot \mathrm{MCO} 3$ & $1.86 \mathrm{E}-11 \cdot \exp (175 / \mathrm{T})$ \\
\hline $\mathrm{MACR}+\mathrm{O} 3$ & $\rightarrow$ & $\begin{array}{l}.8 \cdot \mathrm{CH} 3 \mathrm{COCHO}+.275 \cdot \mathrm{HO} 2+.2 \cdot \mathrm{CO}+.2 \cdot \mathrm{O} 3 \\
+.7 \cdot \mathrm{CH} 2 \mathrm{O}+.215 \cdot \mathrm{OH}\end{array}$ & $4.40 \mathrm{E}-15 \cdot \exp (-2500 / \mathrm{T})$ \\
\hline $\mathrm{MACRO} 2+\mathrm{NO}$ & $\rightarrow$ & $\begin{array}{l}\mathrm{NO} 2+.47 \cdot \mathrm{HO} 2+.25 \cdot \mathrm{CH} 2 \mathrm{O}+.25 \cdot \mathrm{CH} 3 \mathrm{COCHO} \\
+.53 \cdot \mathrm{CH} 3 \mathrm{CO} 3+.53 \cdot \mathrm{GLYALD}+.22 \cdot \mathrm{HYAC}+.22 \cdot \mathrm{CO}\end{array}$ & $2.70 \mathrm{E}-12 \cdot \exp (360 / \mathrm{T})$ \\
\hline $\mathrm{MACRO} 2+\mathrm{NO}$ & $\rightarrow$ & $0.8 \cdot$ ONITR & $1.30 \mathrm{E}-13 \cdot \exp (360 / \mathrm{T})$ \\
\hline $\mathrm{MACRO} 2+\mathrm{NO} 3$ & $\rightarrow$ & $\begin{array}{l}\mathrm{NO} 2+.53 \cdot \mathrm{GLYALD}+.22 \cdot \mathrm{HYAC} \\
+.53 \cdot \mathrm{CH} 3 \mathrm{CO} 3+.25 \cdot \mathrm{CH} 2 \mathrm{O} \\
+.22 \cdot \mathrm{CO}+.25 \cdot \mathrm{CH} 3 \mathrm{COCHO}+.47 \cdot \mathrm{HO} 2\end{array}$ & $2.40 \mathrm{E}-12$ \\
\hline $\mathrm{MACRO} 2+\mathrm{HO} 2$ & $\rightarrow$ & MACROOH & $8.00 \mathrm{E}-13 \cdot \exp (700 / \mathrm{T})$ \\
\hline $\mathrm{MACRO} 2+\mathrm{CH} 3 \mathrm{O} 2$ & $\rightarrow$ & $\begin{array}{l}.73 \cdot \mathrm{HO} 2+.88 \cdot \mathrm{CH} 2 \mathrm{O}+.11 \cdot \mathrm{CO} \\
+.24 \cdot \mathrm{CH} 3 \mathrm{COCHO}+.26 \cdot \mathrm{GLYALD} \\
+.26 \cdot \mathrm{CH} 3 \mathrm{CO} 3+.25 \cdot \mathrm{CH} 3 \mathrm{OH}+.23 \cdot \mathrm{HYAC}\end{array}$ & $5.00 \mathrm{E}-13 \cdot \exp (400 / \mathrm{T})$ \\
\hline $\mathrm{MACRO} 2+\mathrm{CH} 3 \mathrm{CO} 3$ & $\rightarrow$ & $\begin{array}{l}.25 \cdot \mathrm{CH} 3 \mathrm{COCHO}+\mathrm{CH} 3 \mathrm{O} 2+.22 \cdot \mathrm{CO} \\
+.47 \cdot \mathrm{HO} 2+.53 \cdot \mathrm{GLYALD}+.22 \cdot \mathrm{HYAC} \\
+.25 \cdot \mathrm{CH} 2 \mathrm{O}+.53 \cdot \mathrm{CH} 3 \mathrm{CO} 3+\{\mathrm{CO} 2\}\end{array}$ & $1.40 \mathrm{E}-11$ \\
\hline $\mathrm{MACROOH}+\mathrm{OH}$ & $\rightarrow$ & $.5 \cdot \mathrm{MCO} 3+.2 \cdot \mathrm{MACRO} 2+.1 \cdot \mathrm{OH}+.2 \cdot \mathrm{HO} 2$ & $2.30 \mathrm{E}-11 \cdot \exp (200 / \mathrm{T})$ \\
\hline $\mathrm{MCO} 3+\mathrm{NO}$ & $\rightarrow$ & $\mathrm{NO} 2+\mathrm{CH} 2 \mathrm{O}+\mathrm{CH} 3 \mathrm{CO} 3+\{\mathrm{CO} 2\}$ & $5.30 \mathrm{E}-12 \cdot \exp (360 / \mathrm{T})$ \\
\hline $\mathrm{MCO} 3+\mathrm{NO} 3$ & $\rightarrow$ & $\mathrm{NO} 2+\mathrm{CH} 2 \mathrm{O}+\mathrm{CH} 3 \mathrm{CO} 3+\{\mathrm{CO} 2\}$ & $5.00 \mathrm{E}-12$ \\
\hline $\mathrm{MCO} 3+\mathrm{HO} 2$ & $\rightarrow$ & $\begin{array}{l}.25 \cdot \mathrm{O} 3+.25 \cdot \mathrm{CH} 3 \mathrm{COOH} \\
+.75 \cdot \mathrm{CH} 3 \mathrm{COOOH}+.75 \cdot \mathrm{O} 2\end{array}$ & $4.30 \mathrm{E}-13 \cdot \exp (1040 / \mathrm{T})$ \\
\hline $\mathrm{MCO} 3+\mathrm{CH} 3 \mathrm{O} 2$ & $\rightarrow$ & $2 \cdot \mathrm{CH} 2 \mathrm{O}+\mathrm{HO} 2+\{\mathrm{CO} 2\}+\mathrm{CH} 3 \mathrm{CO} 3$ & $2.00 \mathrm{E}-12 \cdot \exp (500 / \mathrm{T})$ \\
\hline $\mathrm{MCO} 3+\mathrm{CH} 3 \mathrm{CO} 3$ & $\rightarrow$ & $2 \cdot\{\mathrm{CO} 2\}+\mathrm{CH} 3 \mathrm{O} 2+\mathrm{CH} 2 \mathrm{O}+\mathrm{CH} 3 \mathrm{CO} 3$ & $4.60 \mathrm{E}-12 \cdot \exp (530 / \mathrm{T})$ \\
\hline $\mathrm{MCO} 3+\mathrm{MCO} 3$ & $\rightarrow$ & $2 \cdot\{\mathrm{CO} 2\}+2 \cdot \mathrm{CH} 2 \mathrm{O}+2 \cdot \mathrm{CH} 3 \mathrm{CO} 3$ & $2.30 \mathrm{E}-12 \cdot \exp (530 / \mathrm{T})$ \\
\hline $\mathrm{MCO} 3+\mathrm{NO} 2+\mathrm{M}$ & $\rightarrow$ & MPAN + M & $1.1 \mathrm{E}-11 \cdot 300 / \mathrm{T} /[\mathrm{M}]$ \\
\hline $\mathrm{MPAN}+\mathrm{M}$ & $\rightarrow$ & $\mathrm{MCO} 3+\mathrm{NO} 2+\mathrm{M}$ & $\begin{array}{l}\mathrm{k}(\mathrm{MCO} 3+\mathrm{NO} 2+\mathrm{M}) \cdot 1.111 \mathrm{E} 28 \\
\cdot \exp (-14000 / \mathrm{T})\end{array}$ \\
\hline $\mathrm{ONITR}+\mathrm{OH}$ & $\rightarrow$ & $\mathrm{HYDRALD}+.4 \cdot \mathrm{NO} 2+\mathrm{HO} 2$ & $4.50 \mathrm{E}-11$ \\
\hline ONITR + NO3 & $\rightarrow$ & HYDRALD + NO2 + HO2 & $1.40 \mathrm{E}-12 \cdot \exp (-1860 / \mathrm{T})$ \\
\hline HYDRALD + OH & $\rightarrow$ & $\mathrm{XO} 2$ & $1.86 \mathrm{E}-11 \cdot \exp (175 / \mathrm{T})$ \\
\hline $\mathrm{XO} 2+\mathrm{NO}$ & $\rightarrow$ & $\begin{array}{l}\mathrm{NO} 2+1.5 \cdot \mathrm{HO} 2+\mathrm{CO}+.25 \cdot \mathrm{CH} 3 \mathrm{COCHO} \\
+.25 \cdot \mathrm{HYAC}+.25 \cdot \mathrm{GLYALD}\end{array}$ & $2.70 \mathrm{E}-12 \cdot \exp (360 / \mathrm{T})$ \\
\hline $\mathrm{XO} 2+\mathrm{NO} 3$ & $\rightarrow$ & $\mathrm{NO} 2+1.5 \cdot \mathrm{HO} 2+\mathrm{CO}+.25 \cdot \mathrm{CH} 3 \mathrm{COCHO}$ & $2.40 \mathrm{E}-12$ \\
\hline $\mathrm{XO} 2+\mathrm{HO} 2$ & $\rightarrow$ & $\mathrm{XOOH}$ & $8.00 \mathrm{E}-13 \cdot \exp (700 / \mathrm{T})$ \\
\hline $\mathrm{XO} 2+\mathrm{CH} 3 \mathrm{O} 2$ & $\rightarrow$ & $\begin{array}{l}.3 \cdot \mathrm{CH} 3 \mathrm{OH}+\mathrm{HO} 2+.7 \cdot \mathrm{CH} 2 \mathrm{O}+.4 \cdot \mathrm{CO}+.1 \cdot \mathrm{HYAC} \\
+.1 \cdot \mathrm{CH} 3 \mathrm{COCHO}+.1 \cdot \mathrm{GLYALD}\end{array}$ & $5.00 \mathrm{E}-13 \cdot \exp (400 / \mathrm{T})$ \\
\hline $\mathrm{XO} 2+\mathrm{CH} 3 \mathrm{CO} 3$ & $\rightarrow$ & $\begin{array}{l}\mathrm{CO}+\mathrm{CH} 3 \mathrm{O} 2+1.5 \cdot \mathrm{HO} 2+\{\mathrm{CO} 2\} \\
+.25 \cdot \mathrm{HYAC}+.25 \cdot \mathrm{CH} 3 \mathrm{COCHO} \\
+.25 \cdot \mathrm{GLYALD}\end{array}$ & $1.30 \mathrm{E}-12 \cdot \exp (640 / \mathrm{T})$ \\
\hline $\mathrm{XOOH}+\mathrm{OH}$ & $\rightarrow$ & $\mathrm{H} 2 \mathrm{O}+\mathrm{XO} 2$ & $1.90 \mathrm{E}-12 \cdot \exp (190 / \mathrm{T})$ \\
\hline $\mathrm{XOOH}+\mathrm{OH}$ & $\rightarrow$ & $\mathrm{H} 2 \mathrm{O}+\mathrm{OH}$ & $\mathrm{T}^{2} \cdot 7.69 \mathrm{E}-17 \cdot \exp (253 / \mathrm{T})$ \\
\hline TOLUENE + OH & $\rightarrow$ & $.25 \cdot \mathrm{CRESOL}+.25 \cdot \mathrm{HO} 2+.7 \cdot \mathrm{TOLO} 2$ & $1.70 \mathrm{E}-12 \cdot \exp (352 / \mathrm{T})$ \\
\hline $\mathrm{CRESOL}+\mathrm{OH}$ & $\rightarrow$ & $\mathrm{XOH}$ & $3.00 \mathrm{E}-12$ \\
\hline $\mathrm{XOH}+\mathrm{NO} 2$ & $\rightarrow$ & $.7 \cdot \mathrm{NO} 2+.7 \cdot \mathrm{BIGALD}+.7 \cdot \mathrm{HO} 2$ & $1.00 \mathrm{E}-11$ \\
\hline $\mathrm{TOLO} 2+\mathrm{NO}$ & $\rightarrow$ & $\begin{array}{l}.45 \cdot \mathrm{GLYOXAL}+.45 \cdot \mathrm{CH} 3 \mathrm{COCHO} \\
+.9 \cdot \mathrm{BIGALD}+.9 \cdot \mathrm{NO} 2+.9 \cdot \mathrm{HO} 2\end{array}$ & $4.20 \mathrm{E}-12 \cdot \exp (180 / \mathrm{T})$ \\
\hline
\end{tabular}


Table 3. Continued.

\begin{tabular}{llll}
\hline Reactants & & Products & Rate \\
\hline $\mathrm{TOLO} 2+\mathrm{HO} 2$ & $\rightarrow$ & TOLOOH & $7.50 \mathrm{E}-13 \cdot \exp (700 / \mathrm{T})$ \\
$\mathrm{TOLOOH}+\mathrm{OH}$ & $\rightarrow$ & TOLO2 & $3.80 \mathrm{E}-12 \cdot \exp (200 / \mathrm{T})$ \\
$\mathrm{C} 10 \mathrm{H} 16+\mathrm{OH}$ & $\rightarrow$ & $\mathrm{TERPO} 2$ & $1.20 \mathrm{E}-11 \cdot \exp (444 / \mathrm{T})$ \\
$\mathrm{C} 10 \mathrm{H} 16+\mathrm{O} 3$ & $\rightarrow$ & $.7 \cdot \mathrm{OH}+\mathrm{MVK}+\mathrm{MACR}+\mathrm{HO} 2$ & $1.00 \mathrm{E}-15 \cdot \exp (-732 / \mathrm{T})$ \\
$\mathrm{C} 10 \mathrm{H} 16+\mathrm{NO} 3$ & $\rightarrow$ & $\mathrm{TERPO} 2+\mathrm{NO} 2$ & $1.20 \mathrm{E}-12 \cdot \exp (490 / \mathrm{T})$ \\
$\mathrm{TERPO} 2+\mathrm{NO}$ & $\rightarrow$ & $.1 \cdot \mathrm{CH} 3 \mathrm{COCH}+\mathrm{HO} 2+\mathrm{MVK}$ & $4.20 \mathrm{E}-12 \cdot \exp (180 / \mathrm{T})$ \\
& & $+\mathrm{MACR}+\mathrm{NO} 2$ & \\
$\mathrm{TERPO} 2+\mathrm{HO} 2$ & $\rightarrow$ & $\mathrm{TERPOOH}$ & $7.50 \mathrm{E}-13 \cdot \exp (700 / \mathrm{T})$ \\
$\mathrm{TERPOOH}+\mathrm{OH}$ & $\rightarrow$ & $\mathrm{TERPO} 2$ & $3.80 \mathrm{E}-12 \cdot \exp (200 / \mathrm{T})$ \\
$\mathrm{SO} 2+\mathrm{OH}$ & $\rightarrow$ & $\mathrm{SO} 4$ & $\mathrm{ko}=3.0 \mathrm{E}-31 \cdot(300 / \mathrm{T})^{3.3} ;$ \\
& & & $\mathrm{ki}=1 . \mathrm{E}-12 ; \mathrm{f}=0.6$ \\
$\mathrm{DMS}+\mathrm{OH}$ & $\rightarrow$ & $\mathrm{SO} 2$ & $9.60 \mathrm{E}-12 \cdot \exp (-234 / \mathrm{T})$ \\
$\mathrm{DMS}+\mathrm{OH}$ & $\rightarrow$ & $.5 \cdot \mathrm{SO} 2+.5 \cdot \mathrm{HO} 2$ & $1.7 \mathrm{E}-42 \cdot \exp (7810 / \mathrm{T}) \cdot[\mathrm{M}] \cdot 0.21 /$ \\
& & & $(1+5.5 \mathrm{E}-31 \cdot \exp (7460 / \mathrm{T}) \cdot[\mathrm{M}] \cdot 0.21)$ \\
$\mathrm{DMS}+\mathrm{NO} 3$ & $\rightarrow$ & $\mathrm{SO} 2+\mathrm{HNO} 3$ & $1.90 \mathrm{E}-13 \cdot \exp (520 / \mathrm{T})$ \\
$\mathrm{NH} 3+\mathrm{OH}$ & $\rightarrow$ & $\mathrm{H} 2 \mathrm{O}$ & $1.70 \mathrm{E}-12 \cdot \exp (-710 / \mathrm{T})$ \\
\hline
\end{tabular}

Table 4. Photolysis reactions.

\begin{tabular}{|c|c|c|}
\hline Reactant & & Products \\
\hline $\mathrm{O} 2+\mathrm{h} v$ & $\rightarrow$ & $2 \cdot \mathrm{O}$ \\
\hline $\mathrm{O} 3+\mathrm{h} v$ & $\rightarrow$ & $\mathrm{O} 1 \mathrm{D}+\mathrm{O} 2$ \\
\hline $\mathrm{O} 3+\mathrm{h} v$ & $\rightarrow$ & $\mathrm{O}+\mathrm{O} 2$ \\
\hline $\mathrm{N} 2 \mathrm{O}+\mathrm{h} v$ & $\rightarrow$ & $\mathrm{O} 1 \mathrm{D}+\mathrm{N} 2$ \\
\hline $\mathrm{NO} 2+\mathrm{h} v$ & $\rightarrow$ & $\mathrm{NO}+\mathrm{O}$ \\
\hline $\mathrm{N} 2 \mathrm{O} 5+\mathrm{h} v$ & $\rightarrow$ & $\mathrm{NO} 2+\mathrm{NO} 3$ \\
\hline $\mathrm{HNO} 3+\mathrm{h} v$ & $\rightarrow$ & $\mathrm{NO} 2+\mathrm{OH}$ \\
\hline $\mathrm{NO} 3+\mathrm{h} v$ & $\rightarrow$ & $.89 \cdot \mathrm{NO} 2+.11 \cdot \mathrm{NO}+.89 \cdot \mathrm{O} 3$ \\
\hline $\mathrm{HO} 2 \mathrm{NO} 2+\mathrm{h} v$ & $\rightarrow$ & $.33 \cdot \mathrm{OH}+.33 \cdot \mathrm{NO} 3+.66 \cdot \mathrm{NO} 2+.66 \cdot \mathrm{HO} 2$ \\
\hline $\mathrm{CH} 3 \mathrm{OOH}+\mathrm{h} v$ & $\rightarrow$ & $\mathrm{CH} 2 \mathrm{O}+\mathrm{HO} 2+\mathrm{OH}$ \\
\hline $\mathrm{CH} 2 \mathrm{O}+\mathrm{h} v$ & $\rightarrow$ & $\mathrm{CO}+2 \cdot \mathrm{HO} 2$ \\
\hline $\mathrm{CH} 2 \mathrm{O}+\mathrm{h} v$ & $\rightarrow$ & $\mathrm{CO}+\mathrm{H} 2$ \\
\hline $\mathrm{H} 2 \mathrm{O} 2+\mathrm{h} v$ & $\rightarrow$ & $2 \cdot \mathrm{OH}$ \\
\hline $\mathrm{CH} 3 \mathrm{CHO}+\mathrm{h} v$ & $\rightarrow$ & $\mathrm{CH} 3 \mathrm{O} 2+\mathrm{CO}+\mathrm{HO} 2$ \\
\hline $\mathrm{POOH}+\mathrm{h} v$ & $\rightarrow$ & $\mathrm{CH} 3 \mathrm{CHO}+\mathrm{CH} 2 \mathrm{O}+\mathrm{HO} 2+\mathrm{OH}$ \\
\hline $\mathrm{CH} 3 \mathrm{COOOH}+\mathrm{h} v$ & $\rightarrow$ & $\mathrm{CH} 3 \mathrm{O} 2+\mathrm{OH}+\{\mathrm{CO} 2\}$ \\
\hline $\mathrm{PAN}+\mathrm{h} v$ & $\rightarrow$ & $.6 \cdot \mathrm{CH} 3 \mathrm{CO} 3+.6 \cdot \mathrm{NO} 2+.4 \cdot \mathrm{CH} 3 \mathrm{O} 2+.4 \cdot \mathrm{NO} 3+.4 \cdot\{\mathrm{CO} 2\}$ \\
\hline MPAN $+h v$ & $\rightarrow$ & $\mathrm{MCO} 3+\mathrm{NO} 2$ \\
\hline $\mathrm{MACR}+\mathrm{h} v$ & $\rightarrow$ & $.67 \cdot \mathrm{HO} 2+.33 \cdot \mathrm{MCO} 3+.67 \cdot \mathrm{CH} 2 \mathrm{O}+.67 \cdot \mathrm{CH} 3 \mathrm{CO} 3+.33 \cdot \mathrm{OH}+0.67 \cdot \mathrm{CO}$ \\
\hline $\mathrm{MVK}+\mathrm{h} v$ & $\rightarrow$ & $.7 \cdot \mathrm{C} 3 \mathrm{H} 6+.7 \cdot \mathrm{CO}+.3 \cdot \mathrm{CH} 3 \mathrm{O} 2+.3 \cdot \mathrm{CH} 3 \mathrm{CO} 3$ \\
\hline $\mathrm{C} 2 \mathrm{H} 5 \mathrm{OOH}+\mathrm{h} v$ & $\rightarrow$ & $\mathrm{CH} 3 \mathrm{CHO}+\mathrm{HO} 2+\mathrm{OH}$ \\
\hline $\mathrm{C} 3 \mathrm{H} 7 \mathrm{OOH}+\mathrm{h} v$ & $\rightarrow$ & $0.82 \cdot \mathrm{CH} 3 \mathrm{COCH} 3+\mathrm{OH}+\mathrm{HO} 2$ \\
\hline $\mathrm{ROOH}+\mathrm{h} v$ & $\rightarrow$ & $\mathrm{CH} 3 \mathrm{CO} 3+\mathrm{CH} 2 \mathrm{O}+\mathrm{OH}$ \\
\hline $\mathrm{CH} 3 \mathrm{COCH} 3+\mathrm{h} v$ & $\rightarrow$ & $\mathrm{CH} 3 \mathrm{CO} 3+\mathrm{CH} 3 \mathrm{O} 2$ \\
\hline $\mathrm{CH} 3 \mathrm{COCHO}+\mathrm{h} v$ & $\rightarrow$ & $\mathrm{CH} 3 \mathrm{CO} 3+\mathrm{CO}+\mathrm{HO} 2$ \\
\hline $\mathrm{XOOH}+\mathrm{h} v$ & $\rightarrow$ & $\mathrm{OH}$ \\
\hline ONITR $+\mathrm{h} v$ & $\rightarrow$ & $\mathrm{HO} 2+\mathrm{CO}+\mathrm{NO} 2+\mathrm{CH} 2 \mathrm{O}$ \\
\hline $\mathrm{ISOPOOH}+\mathrm{h} v$ & $\rightarrow$ & $.402 \cdot \mathrm{MVK}+.288 \cdot \mathrm{MACR}+.69 \cdot \mathrm{CH} 2 \mathrm{O}+\mathrm{HO} 2$ \\
\hline HYAC $+\mathrm{h} v$ & $\rightarrow$ & $\mathrm{CH} 3 \mathrm{CO} 3+\mathrm{HO} 2+\mathrm{CH} 2 \mathrm{O}$ \\
\hline GLYALD + hv & $\rightarrow$ & $2 \cdot \mathrm{HO} 2+\mathrm{CO}+\mathrm{CH} 2 \mathrm{O}$ \\
\hline $\mathrm{MEK}+\mathrm{h} v$ & $\rightarrow$ & $\mathrm{CH} 3 \mathrm{CO} 3+\mathrm{C} 2 \mathrm{H} 5 \mathrm{O} 2$ \\
\hline BIGALD + hv & $\rightarrow$ & $.45 \cdot \mathrm{CO}+.13 \cdot \mathrm{GLYOXAL}+.56 \cdot \mathrm{HO} 2+.13 \cdot \mathrm{CH} 3 \mathrm{CO} 3+0.18 \cdot \mathrm{CH} 3 \mathrm{COCHO}$ \\
\hline GLYOXAL + hv & $\rightarrow$ & $2 \cdot \mathrm{CO}+2 \cdot \mathrm{HO} 2$ \\
\hline $\mathrm{ALKOOH}+\mathrm{h} v$ & $\rightarrow$ & $.4 \cdot \mathrm{CH} 3 \mathrm{CHO}+.1 \cdot \mathrm{CH} 2 \mathrm{O}+.25 \cdot \mathrm{CH} 3 \mathrm{COCH} 3+.9 \cdot \mathrm{HO} 2+.8 \cdot \mathrm{MEK}+\mathrm{OH}$ \\
\hline $\mathrm{MEKOOH}+\mathrm{h} v$ & $\rightarrow$ & $\mathrm{OH}+\mathrm{CH} 3 \mathrm{CO} 3+\mathrm{CH} 3 \mathrm{CHO}$ \\
\hline $\mathrm{TOLOOH}+\mathrm{h} v$ & $\rightarrow$ & $\mathrm{OH}+.45 \cdot \mathrm{GLYOXAL}+.45 \cdot \mathrm{CH} 3 \mathrm{COCHO}+.9 \cdot \mathrm{BIGALD}$ \\
\hline TERPOOH $+\mathrm{h} v$ & $\rightarrow$ & $\mathrm{OH}+.1 \cdot \mathrm{CH} 3 \mathrm{COCH} 3+\mathrm{HO} 2+\mathrm{MVK}+\mathrm{MACR}$ \\
\hline
\end{tabular}


Table 5. Heterogeneous reactions included in MOZART-4, along with the reaction probability $(\gamma)$ and the type of aerosols on which reactions occur.

\begin{tabular}{lllll}
\hline Reaction & & & $\gamma$ & Aerosol \\
\hline $\mathrm{N} 2 \mathrm{O} 5+\mathrm{H} 2 \mathrm{O}$ & $\rightarrow$ & $2 \cdot \mathrm{HNO} 3$ & 0.1 & OC, SO4, NH4NO3, SOA \\
$\mathrm{NO} 3$ & $\rightarrow$ & $\mathrm{HNO} 3$ & 0.001 & OC, SO4, NH4NO3, SOA \\
$\mathrm{NO} 2$ & $\rightarrow$ & $0.5 \cdot \mathrm{NO}+0.5 \cdot \mathrm{HNO}+0.5 \cdot \mathrm{OH}$ & 0.0001 & OC, SO4, NH4NO3, SOA \\
$\mathrm{HO} 2$ & $\rightarrow$ & $0.5 \cdot \mathrm{H} 2 \mathrm{O} 2$ & 0.2 & OC, SO4, NH4NO3, SOA \\
\hline
\end{tabular}

\subsection{Aerosols}

The representation of tropospheric aerosols in MOZART-4 has been extended from the work of Tie et al. $(2001,2005)$, and includes the calculation of sulfate, black carbon, primary organic, secondary organic (SOA), ammonium nitrate, and sea salt (Lamarque et al., 2005). Sulfate aerosols are determined from emissions of $\mathrm{SO}_{2}$ and DMS (Barth et al., 2000). DMS is included to provide an estimate of its source of $\mathrm{SO}_{2}$ as a precursor to aerosols, and other minor products are ignored (Tie et al., 2001). Black carbon and organic carbon aerosols are emitted in a combination of hydrophobic and hydrophilic forms ( $80 \%$ and 50\% hydrophobic, respectively), following Chin et al. (2002). Both black and organic carbon aerosols are converted from hydrophobic to hydrophilic with a rate constant of $7.1 \times 10^{-6} \mathrm{~s}^{-1}$ following Cooke and Wilson (1996), which is equivalent to a time constant of 1.6 days (Horowitz, 2006; Tie et al., 2005). Secondary organic aerosols are linked to the gas-phase chemistry through the oxidation of monoterpenes and toluene as in Chung and Seinfeld (2002). The ammonium nitrate distribution is determined from $\mathrm{NH}_{3}$ emissions and the parameterization of gas/aerosol partitioning by Metzger et al. (2002), which is a set of approximations to the equilibrium constant calculation (Seinfeld, 1986) based on the level of sulfate present.

The uptake of $\mathrm{N}_{2} \mathrm{O}_{5}, \mathrm{HO}_{2}, \mathrm{NO}_{2}$, and $\mathrm{NO}_{3}$ on aerosols is included (Jacob, 2000), with details given in Table 5. Because only the bulk mass is calculated, a lognormal number distribution is assumed for all aerosols to calculate the surface area, using a different geometric mean radius and standard deviation for each type of aerosol, as listed in Table 6 (based on Chin et al., 2002). Sea salt aerosols are included in the model with four size bins $(0.1-0.5,0.5-1.5$, $1.5-5$, and $5-10 \mu \mathrm{m})$ and emissions are calculated online (Mahowald et al., 2006a). The distributions of four sizes of dust $(0.05-0.5,0.5-1.25,1.25-2.5$, and $2.5-5.0 \mu \mathrm{m})$ are set from monthly mean distributions taken from online calculations in the Community Atmosphere Model (CAM) (Mahowald et al., 2006b). Hygroscopic growth of the aerosols is determined from the ambient relative humidity, with different rates for each type of aerosol (Chin et al., 2002). Washout of all aerosols, except hydrophobic black carbon and organic carbon, is set to $20 \%$ of the washout rate of $\mathrm{HNO}_{3}$ (Horowitz, 2006; Tie et al., 2005). Comparison of calculated
Table 6. Bulk aerosol parameters used in calculation of surface area: number distribution mean radius $\left(r_{m}\right)$, geometric standard deviation $\left(\sigma_{g}\right)$ and density.

\begin{tabular}{llll}
\hline Aerosol & $r_{m}(\mathrm{~nm})$ & $\sigma_{g}(\mu \mathrm{m})$ & $\rho\left(\mathrm{g} / \mathrm{cm}^{3}\right)$ \\
\hline CB1, CB2 & 11.8 & 2.00 & 1.0 \\
OC1, OC2 & 21.2 & 2.20 & 1.8 \\
SO4 & 69.5 & 2.03 & 1.7 \\
NH4NO3 & 69.5 & 2.03 & 1.7 \\
SOA & 21.2 & 2.20 & 1.8 \\
\hline
\end{tabular}

Table 7. Approximate matching of MOZART-4 VOCs to other mechanisms.

\begin{tabular}{|c|c|c|c|}
\hline MOZART-4 & SAPRC-99 & RADM2 & CBMZ \\
\hline $\mathrm{C} 2 \mathrm{H} 6$ & ALK1 & ETH & $\mathrm{C} 2 \mathrm{H} 6$ \\
\hline $\mathrm{C} 3 \mathrm{H} 8$ & ALK2 & $\mathrm{HC} 3$ & PAR \\
\hline BIGALK & ALK3+ALK4+ALK5 & HC5 & PAR \\
\hline $\mathrm{C} 2 \mathrm{H} 4$ & ETHE & OL2 & ETH \\
\hline C3H6 & OLE1 & & PAR \\
\hline BIGENE & OLE2 & OLET+OLEI & $\begin{array}{l}\text { OLET+OLEI, } \\
\text { PAR }\end{array}$ \\
\hline TOLUENE & ARO1+ARO2 & $\mathrm{TOL}+\mathrm{XYL}$ & TOL+XYL \\
\hline $\begin{array}{l}\text { ISOP } \\
\text { C10H16 }\end{array}$ & ISOPRENE & ISO & ISOP \\
\hline $\begin{array}{l}\mathrm{CH} 3 \mathrm{OH} \\
\mathrm{C} 2 \mathrm{H} 5 \mathrm{OH}\end{array}$ & $\mathrm{MEOH}$ & & $\mathrm{CH} 3 \mathrm{OH}$ \\
\hline $\mathrm{CH} 2 \mathrm{O}$ & $\mathrm{HCHO}$ & $\mathrm{HCHO}$ & $\mathrm{HCHO}$ \\
\hline СH3CHO & $\mathrm{CCHO}$ & ALD & ALD2 \\
\hline $\mathrm{CH} 3 \mathrm{COOH}$ & & ORA2 & $\mathrm{RCOOH}$ \\
\hline GLYOXAL & & GLY & \\
\hline GLYALD & & ALD & ALD2 \\
\hline $\mathrm{CH} 3 \mathrm{OOH}$ & & $\mathrm{OP} 1$ & $\mathrm{CH} 3 \mathrm{OOH}$ \\
\hline $\mathrm{C} 2 \mathrm{H} 5 \mathrm{OOH}$ & & OP2 & ЕТНООН \\
\hline $\mathrm{CH} 3 \mathrm{COOOH}$ & & PAA & \\
\hline $\mathrm{CH} 3 \mathrm{COCH} 3$ & ACET & KET & AONE \\
\hline HYAC & & KET & AONE \\
\hline $\mathrm{CH} 3 \mathrm{COCHO}$ & & MGLY & MGLY \\
\hline ONIT & & ONIT & ONIT \\
\hline MEK & MEK+PRD2 & KET & AONE \\
\hline MVK & MVK & & ISOPRD \\
\hline MACR & METHACRO & & ISOPRD \\
\hline \multicolumn{4}{|l|}{ MPAN } \\
\hline HYDRALD & & & ISOPRD \\
\hline BIGALD & & & OPEN \\
\hline ISOPNO3 & & & ISOPN \\
\hline ONITR & & & ONIT \\
\hline CRESOL & & CSL & CRES \\
\hline
\end{tabular}


aerosol optical depth (AOD) over ocean to AOD retrievals from the MODIS satellite instrument indicate this is a reasonable washout rate.

\subsection{Photolysis}

A significant improvement in the calculation of photolysis rates in MOZART-4 from MOZART-2 is the use of the online fast-TUV (FTUV) scheme, based on the TUV (Tropospheric Ultraviolet-Visible) model, that takes into account the impact of aerosols and clouds (Tie et al., 2003). The treatment of aerosols in determining photolysis frequencies and aerosol optical depth are from a lookup table based on the Mie calculations used in the NCAR Community Atmosphere Model (CAM3): soot, organic carbon and sea salt are from the Optical Properties of Aerosols and Clouds (OPAC) software package (Hess et al., 1998); ammonium sulfate is based on Tang and Munkelwitz (1994), Toon et al. (1976), and the appendix of Gong et al. (2003); dust optics are from Zender et al. (2003). A lookup table, based on 4-stream calculations from TUV and also used in MOZART-3, can be used instead of FTUV (Kinnison et al., 2007). The lookup table includes explicit calculation of photolysis frequencies for most of the MOZART-4 species, whereas FTUV includes mapping to a subset of the species. This configuration includes the influence of clouds (Chang et al., 1987), but does not account for the impact of aerosols.

\subsection{Albedo}

An improved scheme for the determination of albedo has been implemented, based on satellite observations (Laepple et al., 2005). Monthly snow and non-snow climatologies have been derived from MODIS observations of albedo, and are combined with snow and ice cover information from the model-driving meteorology.

\subsection{Online dry deposition}

Dry deposition velocities can be determined online in the model, based on the resistance-based parameterization of Wesely (1989), Walmsley and Wesely (1996), Wesely and Hicks (2000). The calculation of surface resistances uses the vegetation distribution of Bonan et al. (2003). If the online calculation is not selected at run-time, a monthly mean climatology, based on the same parameterizations using 10 years of NCEP meteorology, is used. In both cases, the deposition velocity calculation has been extended to take into account special cases for $\mathrm{CO}, \mathrm{H}_{2}$ and PAN. In the case of $\mathrm{CO}$ and $\mathrm{H}_{2}$, surface uptake is caused by the oxidation by soil bacteria or enzymes (Yonemura et al., 2000). This has been parameterized using the approach of Sanderson et al. (2003), which defines the deposition velocity by a linear or quadratic function in soil moisture content (or its logarithm), depending on the land cover type. In the case of PAN, new laboratory experiments have indicated a strong uptake of PAN by leaves (Teklemariam and Sparks, 2004). Using the results from that study, we have included a leaf uptake of PAN that is vegetation-dependent, based on Sparks et al. (2003). Results from this parameterization agreed with observations during a field experiment (Turnipseed et al., 2006).

\subsection{Online water calculation}

In MOZART-2 water vapor concentrations were taken from the meteorological fields. However, better agreement with observations of precipitation were found when $\mathrm{H}_{2} \mathrm{O}$ was calculated online from the surface moisture flux and all relevant physics parameters, as implemented in MOZART-3 (Kinnison et al., 2007). This online calculation is a runtime option, and the preferred mode of operation, in MOZART-4.

\subsection{Emissions from vegetation based on MEGAN}

Online calculation of biogenic emissions of isoprene and monoterpenes is based on the Model of Emissions of Gases and Aerosols from Nature (MEGAN) (Guenther et al., 2006) using the implementation described by Pfister et al. (2008a), and in more detail below. While MEGAN parameterizations and emission factor maps have been developed for other species, these have not yet been implemented in MOZART-4. All other biogenic emissions, besides isoprene and monoterpenes, are taken from the POET inventory. These emissions include monthly variation, but are repeated annually for this simulation, without variation from year to year.

Since Guenther et al. (2006) gives a comprehensive description of MEGAN, with various options for particular applications, the details of its implementation in MOZART-4, and suggested for other global models, are included here. The emission factor maps used in MEGAN are updated periodically, as more measurements are made and the algorithms are refined (available from http://bai.acd.ucar.edu/Megan). For the results shown here, version 2.1 of the emission factor maps, the most recent version currently available, are used. In other MOZART-4 studies to date, version 2.0 of the isoprene emission factor maps, and version 1.0 of the monoterpenes emissions, have been used, but they do not differ significantly from version 2.1. The MOZART-4 lumped monoterpene ("C10H16") emissions are the sum of the species alpha-pinene, beta-pinene, limonene, myrcene, ocimene, sabinene, and delta-3-carene in the MEGAN emission factor maps.

The MEGAN formulation also requires global maps of plant functional type (PFT) and the monthly leaf area index (LAI). The PFT and LAI maps used in MOZART-4 are based on AVHRR and MODIS data, as used in the NCAR Community Land Model (CLM) (Lawrence and Chase, 2007). The MEGAN emissions dependency on current and past surface air temperature and solar flux are considered using the model meteorology. Pfister et al. (2008a) illustrate the sensitivity of isoprene emissions to vegetation maps used, showing 


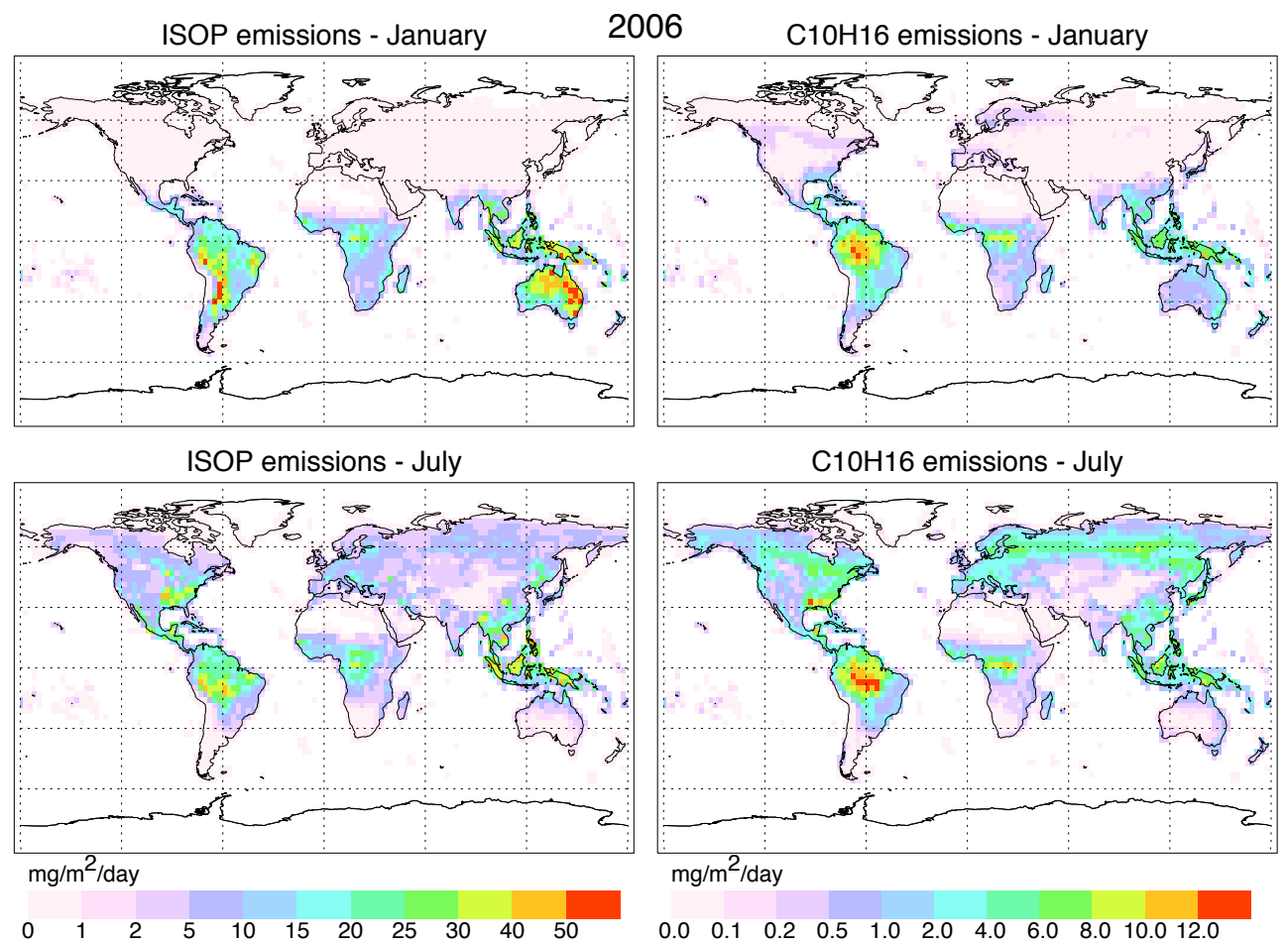

Fig. 1. Emissions of isoprene and monoterpenes for January and July 2006, from online calculation by MEGAN in MOZART-4.

changes of up to $50-100 \%$ for different input maps. It is straight-forward to use other vegetation maps, such as for future climate scenarios, if desired. Using the specified driving variables, the global annual isoprene and monoterpene emissions for the years 2000-2007 are in the range of 530$575 \mathrm{Tg} /$ year and 73-76 Tg/year, respectively. Emissions of isoprene and monoterpenes as calculated in MOZART-4 for January and July 2006 are illustrated in Fig. 1.

\subsubsection{General formulation}

The MEGAN emissions are based on the emission factor EF (the emissions of a compound at standard conditions), the emission activity factor $\gamma$ (a normalized ratio that accounts for deviations from standard conditions), and a factor $\rho$ accounting for the canopy production and loss (assume $=1$ because this is representative of typical conditions).

Global, spatially varying emission factor (EF) maps for each compound (isoprene and monoterpenes for MOZART-4) are provided on a $0.5^{\circ} \times 0.5^{\circ}$ grid for five plant functional types (PFTs): broadleaf trees (btr), needleleaf trees (ntr), shrubs (shr), agricultural crops (crp), and grasses (grs). Landcover maps of PFT fractions per grid box, and leaf area index (LAI) for each month for each PFT are needed. These same maps are used for dry deposition calculations in MOZART-4. 17 PFTs are provided in these maps and must be combined to match the 5 MEGAN PFTs (see Table 8).
Table 8. Plant functional types in Community Land Model (CLM) landcover files and corresponding MEGAN PFTs.

\begin{tabular}{lll}
\hline Index & CLM PFT & MEGAN PFT \\
\hline 1 & desert, ice and ocean & - \\
2 & needleleaf evergreen temperate tree & $\mathrm{ntr}$ \\
3 & needleleaf evergreen boreal tree & $\mathrm{ntr}$ \\
4 & needleleaf deciduous temperate tree & $\mathrm{ntr}$ \\
5 & broadleaf evergreen tropical tree & $\mathrm{btr}$ \\
6 & broadleaf evergreen temperate tree & $\mathrm{btr}$ \\
7 & broadleaf deciduous tropical tree & $\mathrm{btr}$ \\
8 & broadleaf deciduous temperate tree & $\mathrm{btr}$ \\
9 & broadleaf deciduous boreal tree & $\mathrm{btr}$ \\
10 & broadleaf evergreen shrub & $\mathrm{shr}$ \\
11 & broadleaf deciduous temperate shrub & $\mathrm{shr}$ \\
12 & broadleaf deciduous boreal shrub & $\mathrm{shr}$ \\
13 & c3 arctic grass & $\mathrm{grs}$ \\
14 & c3 non-arctic grass & $\mathrm{grs}$ \\
15 & c4 grass & $\mathrm{grs}$ \\
16 & corn & $\mathrm{crp}$ \\
17 & wheat & $\mathrm{crp}$ \\
\hline
\end{tabular}


The emission activity factor depends on the canopy environment $(\mathrm{CE})$, leaf age and soil moisture:

$\gamma=\gamma_{\mathrm{CE}} \cdot \gamma_{\mathrm{age}} \cdot \gamma_{\mathrm{SM}}$

where the canopy environment factor depends on leaf area index (LAI), photosynthetic photon flux density (PPFD) and temperature:

$\gamma_{\mathrm{CE}}=\gamma_{\mathrm{LAI}} \cdot \gamma_{P} \cdot \gamma_{T}$

The soil moisture algorithm described in Guenther et al. (2006) was developed for a specific soil moisture database and wilting point database and may not be appropriate for other soil moisture and wilting point datasets, as pointed out by Müller et al. (2008). Since it is not clear that this algorithm can provide reasonable results with the MOZART driving variables, it has not yet been implemented $\left(\gamma_{\mathrm{SM}}=1\right)$. This may result in an overestimation of global annual isoprene emissions by $\approx 7 \%$ (Guenther et al., 2006).

Thus, for each compound, the emissions $\epsilon$ are determined by:

$\epsilon=\sum_{\mathrm{PFT}} \mathrm{EF}(\mathrm{PFT}) \cdot f_{\mathrm{PFT}} \cdot \gamma_{\mathrm{LAI}} \cdot \gamma_{\mathrm{age}} \cdot \gamma_{P} \cdot \gamma_{T}$

where $f_{\mathrm{PFT}}$ is the fraction of each grid box covered by each PFT, and $\gamma_{\mathrm{LAI}}, \gamma_{\mathrm{age}}, \gamma_{P}$ and $\gamma_{T}$ will vary by compound, and may depend on PFT.

\subsubsection{Isoprene}

The leaf age factor $\left(\gamma_{\text {age }}\right)$ for isoprene emissions from evergreen canopies equals 1 . For deciduous canopies, $\gamma_{\text {age }}$ is a weighted average of emissions from four ages of foliage (new, growing, mature and old):

$\gamma_{\text {age }}=A_{\text {new }} F_{\text {new }}+A_{\text {gro }} F_{\text {gro }}+A_{\text {mat }} F_{\text {mat }}+A_{\text {old }} F_{\text {old }}$

For isoprene, the relative weights of emissions from each canopy type are: $A_{\text {new }}=0.05, A_{\text {gro }}=0.6, A_{\text {mat }}=1.125$ and $A_{\text {old }}=1$. The leaf age fractions $\left(F_{x}\right.$, for new, growing, mature and old states) are calculated according to Eqs. (17-19), and accompanying text, of Guenther et al. (2006). These factors depend on the change in LAI between the current $\left(\mathrm{LAI}_{\mathrm{c}}\right)$ and previous $\left(\mathrm{LAI}_{\mathrm{p}}\right)$ timestep, which for MOZART-4 is a month, based on the time resolution of the LAI maps used. The temperature dependences of Eq. (18) of Guenther et al. (2006) are based on the average temperature of the previous month (cf., Guenther et al., 2006, and corrigendum).

Emission responses to LAI $\left(\gamma_{\mathrm{LAI}}\right)$ variations are estimated according to Eq. (15) of Guenther et al. (2006). The factor accounting for variation in solar radiation $\left(\gamma_{P}\right)$ is a function of the solar angle, the previous month's average abovecanopy PPFD, and the above-canopy PPFD transmission $\phi$ (given by Eqs. (12-13) of Guenther et al., 2006). At low sun angles, $\phi$ can become greater than 1 , so should be limited to a maximum value of 1 .
The temperature dependence for isoprene emissions is determined from:

$\gamma_{T}=E_{\mathrm{opt}} \cdot C_{T 2} \cdot \exp \left(C_{T 1} \cdot x\right) /\left(C_{T 2}-C_{T 1} \cdot\left(1-\exp \left(C_{T 2} \cdot x\right)\right)\right)$

where $x=\left(T_{\mathrm{p}}-T_{\mathrm{opt}}\right) /\left(T_{\mathrm{p}} \cdot T_{\mathrm{opt}} \cdot 0.00831\right), \quad C_{T 1}=80$ and $C_{T 2}=200, T_{\mathrm{opt}}=313+0.6 \cdot\left(T_{\mathrm{p}}-T_{\mathrm{clim}}\right), E_{\mathrm{opt}}=1.75 \cdot \exp (0.08 \cdot$ $\left.\left(T_{\mathrm{p}}-T_{\mathrm{clim}}\right)\right)$, with $T_{\mathrm{p}}$ being the average temperature of the previous month and $T_{\text {clim }}$ the daily average climatological value $(297 \mathrm{~K})$. To be consistent with the dependence of $\gamma_{\text {age }}$ on the previous monthly average of LAI, $T_{\mathrm{p}}$ refers to the monthly average temperature, as opposed to the previous hourly temperature used in Guenther et al. (2006).

\subsubsection{Monoterpenes}

MOZART-4 includes a simplified implementation of monoterpene emissions that does not depend on sunlight $\left(\gamma_{P}=1\right)$ or leaf age $\left(\gamma_{\mathrm{age}}=1\right)$, and has simple LAI and temperature factors, with no dependence on past solar radiation:

$\gamma_{\mathrm{LAI}}=0.2 \cdot \mathrm{LAI}$

$\gamma_{T}=\exp \left(0.09 \cdot\left(T_{\mathrm{p}}-T_{\mathrm{std}}\right)\right)$

where $T_{\mathrm{p}}$ is the average temperature of the previous month and $T_{\text {std }}=303.15$.

\subsection{Soil and lightning NO emissions}

Soil NO emissions are a combination of interactive natural emissions (Yienger and Levy, 1995) and fertilizer use (Bouwman et al., 2002). The soil NO emissions are highly dependent on the degree of soil dryness; in order to keep track of this quantity, we have added to the model a bucketstyle parameterization of soil moisture, taking into account the model-calculated precipitation and input latent heat flux, as described by Yienger and Levy (1995). If the soil moisture is not available in the meteorological input dataset, then the model uses a 10-year climatology of monthly-averaged soil moisture from NCEP/NCAR-reanalysis meteorological fields. On average, for late 20th century conditions, the global annual average of soil NO emissions amount to approximately $7 \mathrm{Tg}-\mathrm{N} / \mathrm{year}$, with about half of that due to the effect of fertilization (see Yienger and Levy, 1995).

The lightning parameterization differs slightly from that used in MOZART-2 (Horowitz et al., 2003). The lightning strength still depends on cloud top height, with a stronger dependence over land than ocean (Price et al., 1997). The definition of ocean grid boxes has been refined to include only boxes surrounded by ocean, so that the land parameterization is extended one grid box beyond the continents (Price and Rind, 1992). Flash frequency is determined by area, not grid box. The vertical distribution of NO emissions has been modified from that given by Pickering et al. (1998), to have a reduced proportion of the emissions emitted near the surface, 
similar to that used by DeCaria et al. (2006). In addition, the strength of intra-cloud (IC) lightning strikes is assumed to be equal to cloud-to-ground strikes, as recommended by Ridley et al. (2005). The annual lightning emissions for 2006 from MOZART-4 driven by NCEP/GFS are shown in Fig. 2.

\subsection{Surface boundary conditions}

For some long-lived species such as $\mathrm{CH}_{4}, \mathrm{H}_{2}$, and $\mathrm{N}_{2} \mathrm{O}$, their tropospheric concentrations are known more accurately than their emissions. Therefore, MOZART-4 simulations are generally run using fixed lower boundary conditions constrained by observations, instead of direct emissions for these species. $\mathrm{CH}_{4}$ monthly zonal averages are based on the measurements by NOAA/ESRL Global Monitoring Division Cooperative Air Sampling Network (Dlugokencky et al., 2005, 2008). $\mathrm{H}_{2}$ is set to $530 \mathrm{ppbv}$, based on global average observations made by NOAA/ESRL/GMD for 1993-2003 (Novelli et al., 1999). $\mathrm{N}_{2} \mathrm{O}$ concentrations are taken from IPCC (2000).

\subsection{Upper boundary conditions}

Mixing ratios of several species $\left(\mathrm{O}_{3}, \mathrm{NO}_{\mathrm{x}}, \mathrm{HNO}_{3}\right.$, $\mathrm{N}_{2} \mathrm{O}_{5}, \mathrm{CO}, \mathrm{CH}_{4}$ ) are constrained in the stratosphere since MOZART-4 does not have complete stratospheric chemistry. These mixing ratios have been updated to zonal means from a MOZART-3 simulation. Model concentrations are set to the climatology values above $50 \mathrm{hPa}$, and relaxed to the climatology with a 10-day time scale down to the tropopause. The primary change from the climatology used in MOZART2 is a reduction in the $\mathrm{NO}_{\mathrm{x}}$ and $\mathrm{HNO}_{3}$ mixing ratios in the lower stratosphere. $\mathrm{O}_{3}$ is still constrained to observations (from satellite and ozonesondes), as in MOZART-2 (Horowitz et al., 2003). A stratospheric aerosol surface area density climatology (SPARC, 2006) has been included in MOZART-4 so as to more accurately represent the fast $\mathrm{NO}_{\mathrm{y}}$ partitioning in the lower stratosphere. The gas-phase and heterogenous reactions operate at all model levels, without any additional parameterizations for stratospheric chemistry. It should be noted that MOZART-4 is generally not suitable for studies of the dynamics and detailed structure of the upper troposphere and stratosphere due to these constraints. For example, the impact of lightning and aircraft emissions in the lower stratosphere may not be accurately represented (Grewe et al., 2002).

Reanalysis meteorological datasets generally result in a stratospheric flux that is too strong in offline chemical transport models (Van Noije et al., 2004, and references therein), resulting in errors in the tropospheric ozone budget and ozone mixing ratios that are too high in the upper troposphere. This can be the case for MOZART-4 when driven by NCEP/NCAR reanalyses (Kalnay et al., 1996; Kistler et al., 2001) and ECMWF reanalyses (ERA-40) (Simmons and Gibson, 2000). Preliminary simulations with these re-
Annual Total Lightning NO Emissions - 2006

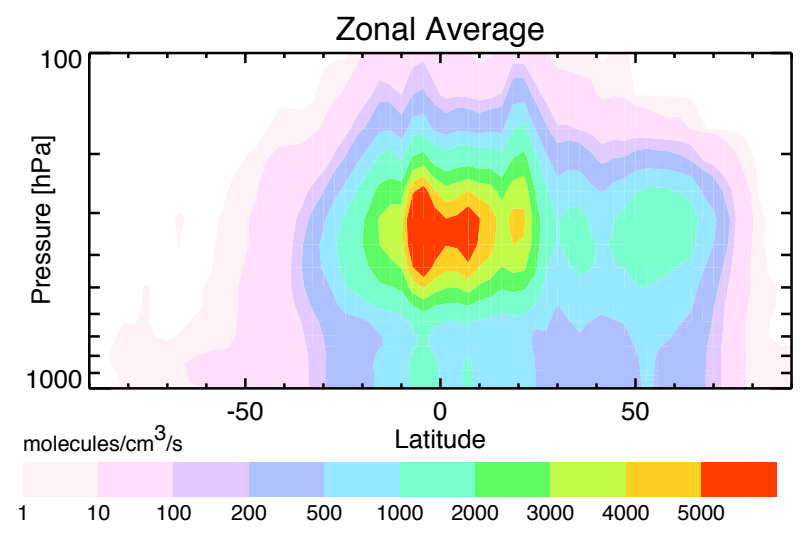

Total Column

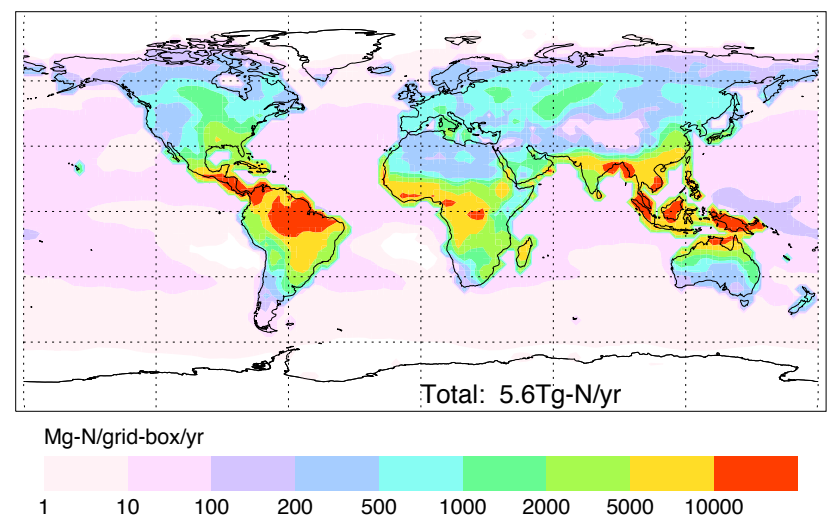

Fig. 2. Emissions of NO from lightning for 2006. Top panel shows the zonal average vertical distribution for the annual average. Bottom panel shows the total column annual total (in $\mathrm{Mg}$ N/gridbox/yr).

analyses resulted in ozone mixing ratios in the upper troposphere that were much higher than observations, such as ozonesondes.

In order to constrain the stratospheric flux of ozone independently of the meteorological dataset used, the synthetic ozone (SYNOZ) representation is used (McLinden et al., 2000). SYNOZ is a tracer with a specified source region (30S-30N, 10-70 hPa) and production rate (400-500 Tg/yr); after production, SYNOZ is advected as a passive tracer. In order to provide a tropospheric sink, the SYNOZ mixing ratio is relaxed to $25 \mathrm{ppbv}$ below $500 \mathrm{hPa} . \mathrm{O}_{3}$ is set to $\mathrm{SYNOZ}$ above the tropopause, if $\mathrm{SYNOZ}>100 \mathrm{ppbv}$; this limits the overwriting of the modeled ozone field by SYNOZ to the lower stratosphere. Because SYNOZ does not provide a realistic distribution of ozone in the stratosphere, an additional tracer, named O3RAD, is relaxed to the ozone climatology (mentioned above) in the stratosphere and to the model calculated ozone in the troposphere; this additional tracer is only used for photolysis calculations. To illustrate the relationship between the SYNOZ, O3RAD and O3 variables, a monthly 


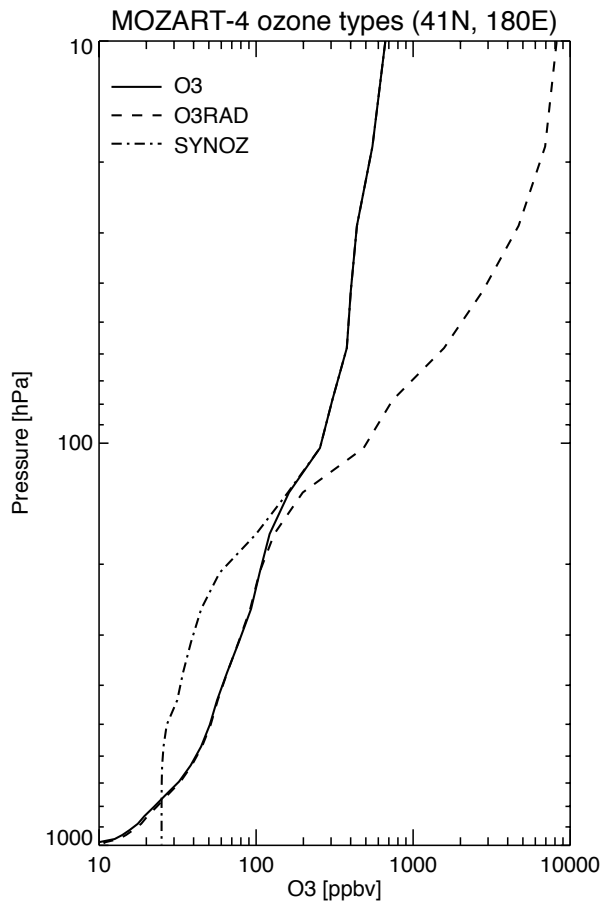

Fig. 3. Example profiles of the three ozone variables (O3, O3RAD and SYNOZ) used when including the SYNOZ parameterization. Shown is a monthly average profile for July 2006 at a point over the Pacific Ocean.

mean profile is shown in Fig. 3. When SYNOZ is used, the $\mathrm{O} 3$ variable is appropriate only for the troposphere, and should not be used for studies of the tropopause region. For simulations using the NCEP/GFS analyses it was found not to be necessary to use SYNOZ to get accurate ozone amounts in the upper troposphere, probably due to the higher vertical resolution than the NCAR reanalysis (42 levels instead of 28) (Pfister et al., 2008b).

\section{Previous MOZART-4 applications}

MOZART-4 has already been used in several studies where it has been shown to reproduce observations well. Detailed comparisons have been made of MOZART-4 results to the aircraft, ozonesonde and ground-based observations from the International Consortium for Atmospheric Research on Transport and Transformation (ICARTT) during Summer 2004 (Pfister et al., 2005, 2006; Lapina et al., 2006; Horowitz et al., 2007; Pfister et al., 2008b). MOZART-4 simulations have been used for boundary conditions in regional models (Tang et al., 2009; Fast et al., 2009; Mena-Carrasco et al., 2009). Significant improvement in regional model results have been found when time-varying chemical boundary conditions, such as from MOZART-4, are used (e.g., Tang et al., 2009).
MOZART-4 results have also been included in multimodel comparisons, such as the study coordinated by the European Union project Atmospheric Composition Change: the European Network of excellence (ACCENT; http://www. accent-network.org) (Dentener et al., 2006; Stevenson et al., 2006; Shindell et al., 2006). Chemical forecasts have been produced with MOZART-4 and used in flight planning activities for aircraft experiments such as the NSF Megacities Impact on Regional and Global Environment (MIRAGE) Mexico City campaign in March 2006 (Fast et al., 2007), and the NASA Intercontinental Chemical Transport Experiment (INTEX-B) in April-May 2006 (Singh et al., 2009). Detailed comparisons of MOZART- 4 results to the MIRAGE and INTEX-B experiments are given in Emmons et al. (2010) and Pfister et al. (2009).

\section{MOZART-4 simulation of 2000-2007}

A simulation of 2000-2007 is presented here to illustrate the capabilities of MOZART-4 and for its evaluation. This simulation was driven by NCEP/NCAR-reanalysis meteorological fields, at a horizontal resolution of $2.8^{\circ} \times 2.8^{\circ}$ (28 levels). The artificial stratospheric ozone mechanism, SYNOZ, was used. Dry deposition velocities, water vapor concentrations, photolysis rates using FTUV, and biogenic emissions for soil NO, and for isoprene and monoterpenes from MEGAN, were calculated online using the new interactive schemes described above.

\subsection{Emissions}

A number of emissions inventories are suitable for use in MOZART-4, depending on the application. For this overview, several inventories have been used to cover all of the species and emissions types. These inventories, as used in MOZART-4, are provided with the MOZART-4 source code (http://cdp.ucar.edu).

The majority of the anthropogenic emissions used for this simulation came from the POET (Precursors of Ozone and their Effects in the Troposphere) database for 2000 (Granier et al., 2005; Olivier et al., 2003), which includes anthropogenic emissions (from fossil fuel and biofuel combustion) based on the EDGAR-3 inventory (Olivier and Berdowski, 2001). The anthropogenic emissions (from fossil fuel and biofuel combustion) of black and organic carbon determined for 1996 are from Bond et al. (2004). For $\mathrm{SO}_{2}$ and $\mathrm{NH}_{3}$, anthropogenic emissions are from the EDGAR-FT2000 and EDGAR-2 databases, respectively (http://www.mnp.nl/edgar/). For Asia, these inventories have been replaced by the Regional Emission inventory for Asia (REAS) with the corresponding annual inventory for each year simulated (Ohara et al., 2007). Aircraft emissions of $\mathrm{NO}, \mathrm{CO}$ and $\mathrm{SO}_{2}$ from scheduled, charter, general aviation and military traffic for 1999 are also included (Baughcum 
Table 9. Emissions totals by category for 2006 in $\mathrm{Tg}$ (species)/year.

\begin{tabular}{lrrrrrrr}
\hline Species & Anthro & Fires & $\begin{array}{r}\text { Bio- } \\
\text { genic }\end{array}$ & Soil & Ocean & Volcano & Total \\
\hline NO & 77.3 & 10.8 & 0.0 & 11.7 & 0.0 & 0.0 & 99.8 \\
CO & 642.0 & 388.9 & 159.9 & 0.0 & 19.9 & 0.0 & 1210.7 \\
C2H6 & 7.8 & 3.1 & 1.0 & 0.0 & 0.0 & 0.0 & 11.9 \\
C3H8 & 8.4 & 0.6 & 2.0 & 0.0 & 0.0 & 0.0 & 11.0 \\
C2H4 & 7.3 & 5.8 & 5.0 & 0.0 & 0.0 & 0.0 & 18.1 \\
C3H6 & 3.0 & 1.8 & 1.0 & 0.0 & 0.0 & 0.0 & 5.8 \\
BIGALK & 77.6 & 1.4 & 0.0 & 0.0 & 0.0 & 0.0 & 78.9 \\
BIGENE & 7.6 & 1.4 & 0.0 & 0.0 & 0.0 & 0.0 & 9.1 \\
TOLUENE & 31.5 & 2.8 & 0.0 & 0.0 & 0.0 & 0.0 & 34.3 \\
ISOP & 0.0 & 0.0 & 469.6 & 0.0 & 0.0 & 0.0 & 469.6 \\
C10H16 & 0.0 & 0.0 & 90.7 & 0.0 & 0.0 & 0.0 & 90.7 \\
CH3OH & 0.4 & 7.6 & 229.6 & 0.0 & 0.0 & 0.0 & 237.6 \\
C2H5OH & 5.4 & 0.4 & 0.0 & 0.0 & 0.0 & 0.0 & 5.8 \\
CH2O & 1.1 & 2.9 & 0.0 & 0.0 & 0.0 & 0.0 & 4.0 \\
CH3CHO & 2.2 & 5.4 & 0.0 & 0.0 & 0.0 & 0.0 & 7.6 \\
CH3COCH3 & 0.3 & 2.4 & 24.4 & 0.0 & 0.0 & 0.0 & 27.2 \\
MEK & 1.4 & 5.2 & 0.0 & 0.0 & 0.0 & 0.0 & 6.6 \\
SO2 & 139.4 & 2.3 & 0.0 & 0.0 & 0.0 & 9.6 & 151.4 \\
DMS & 0.0 & 0.0 & 0.0 & 0.0 & 27.4 & 0.0 & 27.4 \\
NH3 & 51.2 & 5.7 & 0.0 & 2.4 & 8.2 & 0.0 & 67.5 \\
CB1+CB2 & 5.0 & 2.6 & 0.0 & 0.0 & 0.0 & 0.0 & 7.6 \\
OC1+OC2 & 16.9 & 21.3 & 0.0 & 0.0 & 0.0 & 0.0 & 38.1 \\
\hline & & & & & & & \\
\hline
\end{tabular}

et al., 1996, 1998; Mortlock and Alstyne, 1998; Sutkus et al., 2001), and have global annual totals of $0.63 \mathrm{Tg} / \mathrm{yr}(1.35$ $\mathrm{TgN} / \mathrm{yr}$ ) for $\mathrm{NO}, 1.70 \mathrm{Tg} / \mathrm{yr}$ for $\mathrm{CO}$ and $0.16 \mathrm{Tg} / \mathrm{yr}$ for $\mathrm{SO}_{2}$. Only the Asian emissions from REAS vary each year, all other emissions are repeated annually for each year of simulation.

Monthly average biomass burning emissions for each year are from the Global Fire Emissions Database, version 2 (GFED-v2), which is currently available for 1997-2007 (van der Werf et al., 2006). For species not provided in GFED (e.g., individual volatile organic compounds as specified in MOZART-4, $\mathrm{SO}_{2}$, and $\mathrm{NH}_{3}$ ), emissions are determined by scaling the GFED $\mathrm{CO}_{2}$ emissions by the emission factors of Andreae and Merlet (2001) and updates (Granier et al., 2005), using the vegetation classification provided with GFED. While MOZART-4 provides the capability to use vertically distributed emissions (such as for biomass burning), for these simulations, all emissions, except aircraft and lightning, are emitted at the surface.

Emissions of isoprene and monoterpenes from vegetation, and NO from soil and lightning, are calculated online, as described above. The DMS emissions are monthly means from the marine biogeochemistry model HAMOCC5, representative of the year 2000 (Kloster et al., 2006). $\mathrm{SO}_{2}$ emissions from continuously outgassing volcanoes are from the GEIAv1 inventory (Andres and Kasgnoc, 1998).

The surface emissions totals for 2006, separated by category, are shown in Table 9, and summarized for each year in Table 10. NO emissions from lightning are included in Table 10.
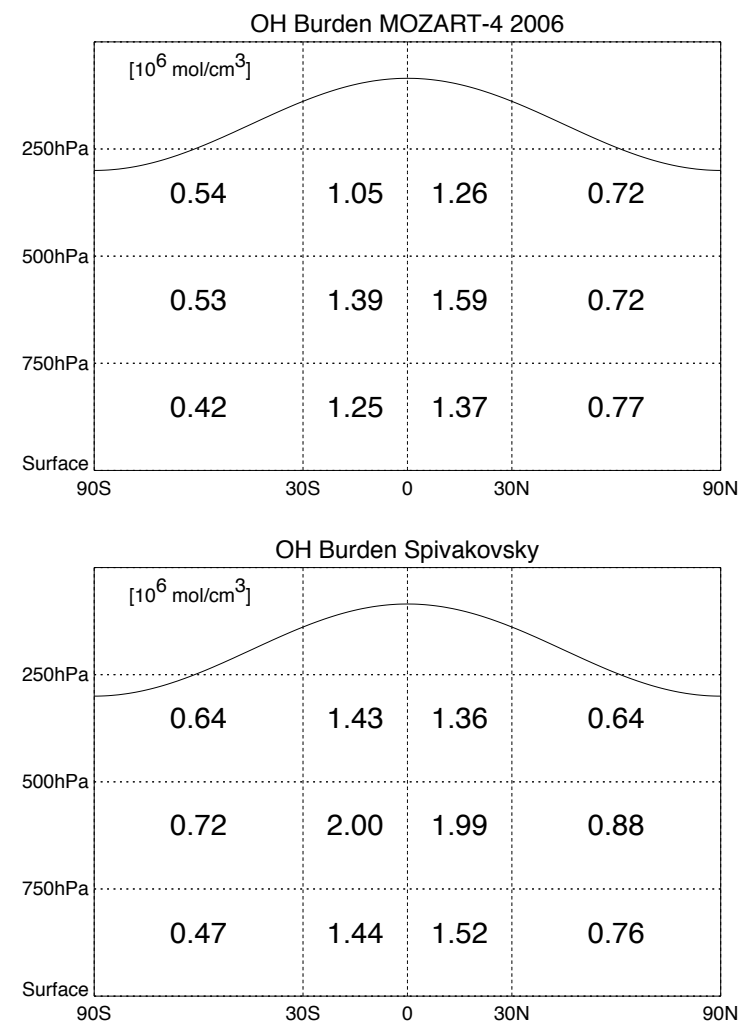

Fig. 4. OH burden from MOZART-4 compared to the climatology of Spivakovsky et al. (2000).

\section{Model evaluation}

To illustrate the capability of MOZART-4 to reproduce the true atmosphere, a few examples of the evaluation of the model through comparisons with a standard $\mathrm{OH}$ climatology, network ground sites, ozonesondes and satellite observations of $\mathrm{CO}$ and aerosol optical depth are shown.

\subsection{Hydroxyl radical}

Simulated $\mathrm{OH}$ is compared to the climatology of Spivakovsky et al. (2000) in Fig. 4 in terms of the annual airmass-weighted burden, as recommended by Lawrence et al. (2001). For this MOZART-4 simulation, OH is somewhat lower than the climatology, and gives a methane lifetime of about 10.5 years (2000-2007 global average, for the troposphere, altitudes below $100 \mathrm{hPa}$ ), which is on the high side of previous model estimates. For example, the MOZART-2 standard simulation had a methane lifetime of 9.4 years (Horowitz et al., 2003), and the MATCH simulations of Lawrence et al. (2001) gave lifetimes between 7.8 and 10.3 years. The MOZART-4 distribution shows less of an increase with altitude from the lower troposphere to the upper troposphere than the climatology, but is similar to the shape of the MATCH simulations (Lawrence et al., 2001). This could indicate limitations in the climatology, which is 
Table 10. Emissions totals for 2000-2007 in Tg(species)/year.

\begin{tabular}{lrrrrrrrr}
\hline Species & 2000 & 2001 & 2002 & 2003 & 2004 & 2005 & 2006 & 2007 \\
\hline NO & 92.3 & 94.0 & 96.5 & 98.3 & 98.9 & 99.8 & 99.8 & 101.3 \\
Lght-NO [TgN/yr] & 4.8 & 5.0 & 4.6 & 4.8 & 4.7 & 5.3 & 5.6 & 5.8 \\
CO & 1114.5 & 1146.8 & 1211.4 & 1204.1 & 1222.1 & 1225.7 & 1213.2 & 1235.1 \\
C2H6 & 10.9 & 11.1 & 11.7 & 11.4 & 11.9 & 12.0 & 11.9 & 12.0 \\
C3H8 & 10.5 & 10.6 & 10.8 & 10.8 & 10.9 & 10.9 & 11.0 & 11.1 \\
C2H4 & 16.4 & 16.9 & 17.8 & 17.4 & 18.2 & 18.4 & 18.1 & 18.4 \\
C3H6 & 5.3 & 5.5 & 5.8 & 5.8 & 5.8 & 5.9 & 5.8 & 5.9 \\
BIGALK & 75.7 & 76.2 & 76.8 & 77.4 & 78.0 & 78.5 & 79.1 & 79.7 \\
BIGENE & 8.1 & 8.2 & 8.7 & 8.8 & 8.9 & 9.0 & 9.1 & 9.1 \\
TOLUENE & 31.6 & 32.1 & 33.0 & 33.4 & 33.6 & 34.0 & 34.3 & 34.8 \\
ISOP & 450.7 & 463.7 & 481.5 & 472.3 & 473.3 & 488.4 & 469.6 & 466.9 \\
C10H16 & 88.1 & 89.6 & 90.2 & 90.0 & 89.2 & 91.7 & 90.7 & 90.6 \\
CH3OH & 236.8 & 237.2 & 238.1 & 237.6 & 238.1 & 238.0 & 237.6 & 238.0 \\
C2H5OH & 5.8 & 5.8 & 5.9 & 5.8 & 5.8 & 5.8 & 5.8 & 5.8 \\
CH2O & 3.2 & 3.3 & 4.1 & 4.1 & 3.9 & 3.9 & 4.0 & 3.9 \\
CH3CHO & 6.9 & 7.3 & 8.0 & 7.8 & 7.9 & 7.9 & 7.7 & 8.0 \\
CH3COCH3 & 26.9 & 27.1 & 27.4 & 27.2 & 27.4 & 27.4 & 27.2 & 27.4 \\
MEK & 5.8 & 6.2 & 6.9 & 6.6 & 6.8 & 6.8 & 6.6 & 6.9 \\
SO2 & 141.3 & 143.6 & 146.9 & 151.8 & 151.6 & 151.5 & 151.5 & 151.4 \\
DMS & 27.4 & 27.4 & 27.4 & 27.4 & 27.4 & 27.4 & 27.4 & 27.4 \\
NH3 & 65.4 & 65.9 & 66.6 & 66.4 & 67.0 & 67.5 & 67.7 & 68.5 \\
CB1 & 5.7 & 5.9 & 6.1 & 6.0 & 6.2 & 6.2 & 6.1 & 6.2 \\
CB2 & 1.4 & 1.5 & 1.5 & 1.5 & 1.5 & 1.5 & 1.5 & 1.6 \\
OC1 & 17.5 & 18.0 & 20.1 & 20.0 & 19.3 & 19.2 & 19.1 & 19.4 \\
OC2 & 17.5 & 18.0 & 20.1 & 20.0 & 19.3 & 19.2 & 19.1 & 19.4 \\
SA1 & 64.8 & 65.7 & 66.0 & 65.8 & 65.8 & 65.5 & 68.8 & 82.7 \\
SA2 & 708.3 & 718.4 & 722.0 & 719.4 & 719.3 & 716.2 & 752.1 & 904.1 \\
SA3 & 1667.0 & 1690.7 & 1699.2 & 1693.1 & 1693.0 & 1685.8 & 1770.2 & 2127.8 \\
SA4 & 953.3 & 966.8 & 971.7 & 968.2 & 968.2 & 964.0 & 1012.3 & 1216.8 \\
\hline
\end{tabular}

derived from observations, as much as errors in the model simulation. MOZART-4 configurations with different meteorology and emissions could result in different $\mathrm{OH}$ distributions, and consequently different $\mathrm{CH}_{4}$ lifetimes.

\subsection{Carbon monoxide}

Comparison of carbon monoxide (CO) in MOZART-4 to the NOAA ESRL Global Monitoring Division surface sites (Novelli et al., 2003) is shown in Fig. 5. The model generally reproduces the seasonal cycle well, and captures much of the interannual variability. The model results are interpolated to the pressure altitude of the observation sites, so the mountain sites of Niwot Ridge (Colorado), Tenerife (Canary Islands), and Mauna Loa (Hawaii) are comparisons of free tropospheric air, generally not directly influenced by surface emissions.

The mid to high southern latitudes are over-estimated by the model, perhaps due to an over-estimation of biogenic or biomass burning emissions (since anthropogenic emissions are relatively small in the Southern Hemisphere), or too low simulated $\mathrm{OH}$ concentrations (as indicated in Fig. 4). The large Australian fires at the start of the year in 2003 and 2007 show up strongly in the model in the Cape Grim comparison, due to the large model grid box that encompasses both Cape Grim and southern Australia. The underestimate of $\mathrm{CO}$ at Tenerife and Mauna Loa during Spring 2003 is probably due to the strong biomass burning in Siberia during that time.

The global distributions of $\mathrm{CO}$ from the Measurements of Pollution In The Troposphere instrument on the Terra satellite are a valuable data set for model evaluation (e.g., Shindell et al., 2006). The recently released MOPITT V4 retrievals (Deeter et al., 2010) are used for comparison to the MOZART-4 simulated CO. Figure 6 shows an example comparison of the total column CO for May 2003. The top panel shows the column average mixing ratio directly from the model output and the middle panel shows the MOPITT column retrieval, expressed as average mixing ratio. To properly compare these two products, the averaging kernel and a priori profile associated with each MOPITT retrieval must be applied to the model profiles, as described in Emmons et al. (2009). The model appears to overestimate the CO produced 

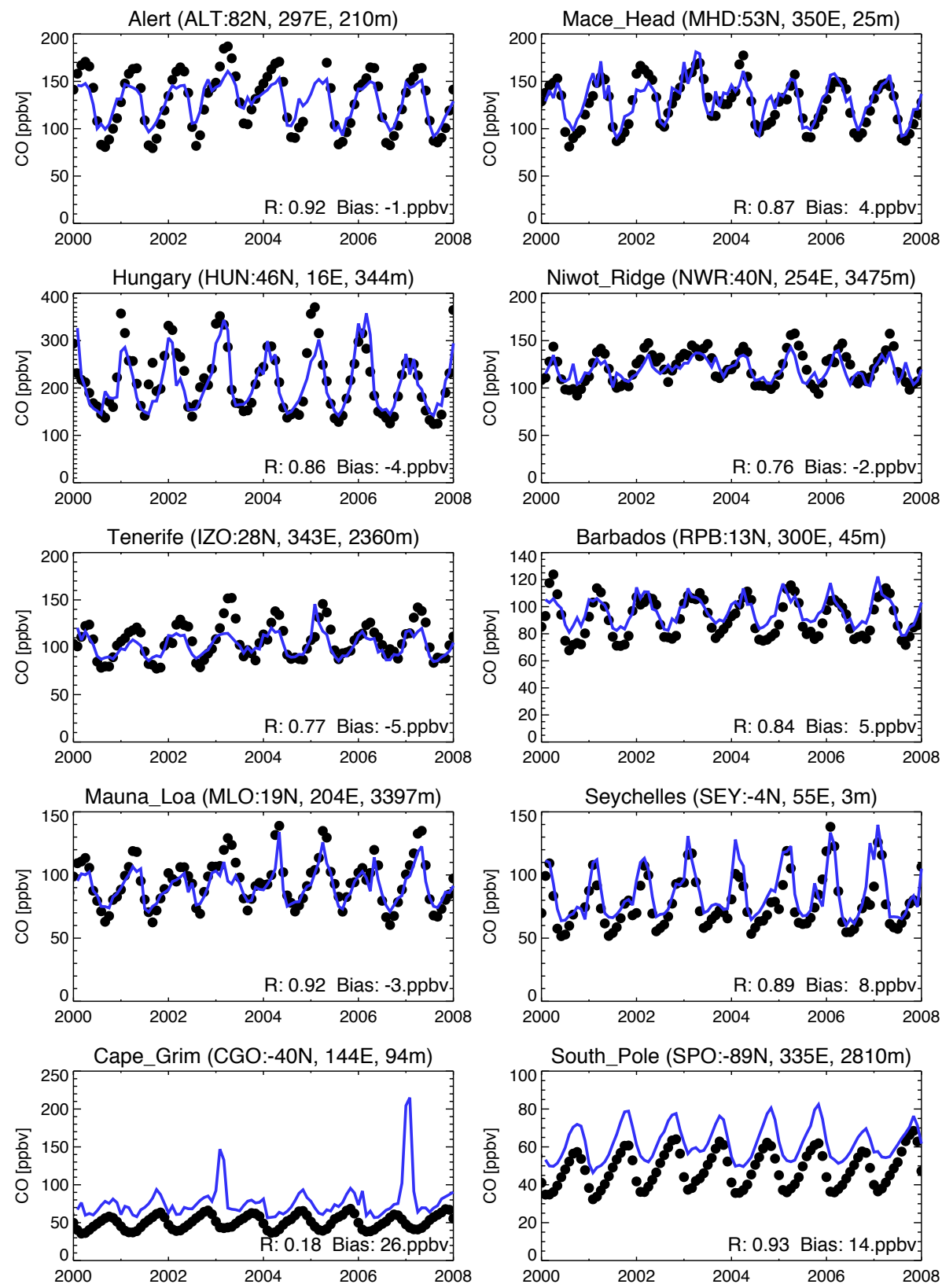

Fig. 5. Comparison of MOZART-4 CO (blue line) to NOAA GMD surface CO measurements (black dots) at selected network sites. MOZART-4 results correspond to the pressure altitude of each observation site. Measurements are monthly averages of approximately weekly samples, while model results are averages of the complete month.

by the large fires in Siberia during this month. Other regions of biomass burning, such as the Yucatan peninsula in Mexico, and central Africa, are also a bit high in MOZART-4. This may be due to too high fire emissions from the GFED-v2 inventory, or may be an artifact of having all the emissions released into the model at the surface, instead of vertically distributed, which would be more realistic in many cases. Outside of Siberia, MOZART-4 underestimates the Northern Hemisphere distribution (by 15-30 ppbv).
To evaluate the model in greater detail, comparisons for 2002-2007 are shown in Fig. 7 for various regions. The monthly mean retrievals and monthly mean model results (with MOPITT averaging kernels and a priori applied) have been averaged over various continental regions. The error bars on the MOPITT observations, and the ranges indicated for the model results, indicate the range of the data (10th to 90th percentiles) over the region. In general the agreement between MOZART-4 and MOPITT is quite good, as 

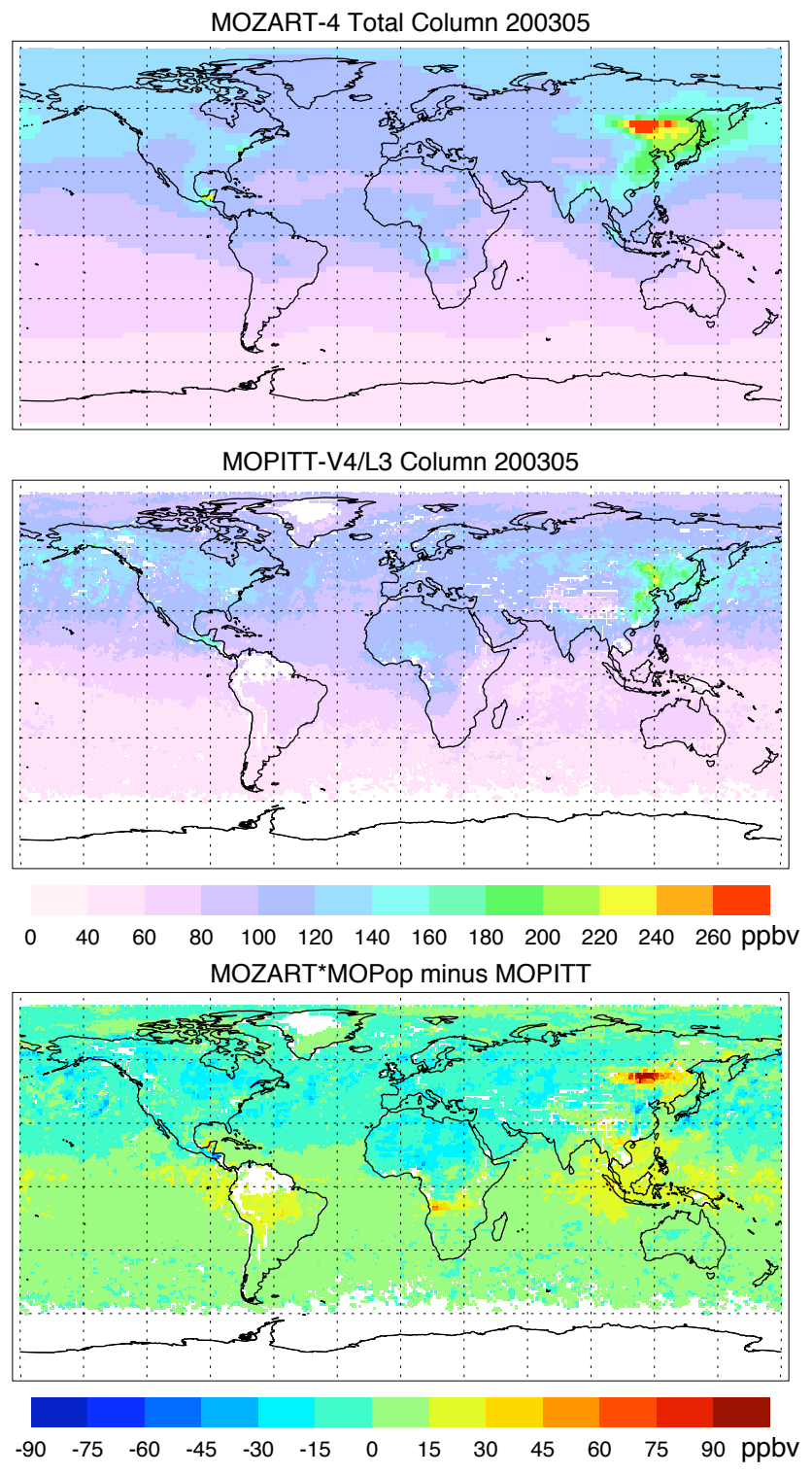

Fig. 6. Evaluation of MOZART-4 CO with MOPITT V4 CO total column densities; top: MOZART-4 CO total column average mixing ratio; middle: MOPITT CO total column retrieval as average mixing ratio; bottom: difference between MOZART-4 CO, transformed with the MOPITT operator (a priori and averaging kernels), and MOPITT CO, for May 2003.

indicated by the correlation coefficients and mean biases (as indicated in Fig. 7). In the Northern Hemisphere, the model appears to be a bit low, indicating perhaps the anthropogenic emissions are underestimated, as that is the dominant source on average. Since MOPITT is most sensitive to the free troposphere, the differences in these comparisons could be a combination of transport (including convection and boundary layer venting) and emissions errors in the model. In the regions with significant biomass burning impact (such as Southeast Asia, Indonesia, and S. America), the model
Table 11. Locations of ozonesondes used in Fig. 7.

\begin{tabular}{|c|c|c|c|}
\hline Station & Location & Station & Location \\
\hline \multicolumn{2}{|c|}{$90 \mathrm{~S}-30 \mathrm{~S}$} & \multicolumn{2}{|c|}{$30 \mathrm{~N}-90 \mathrm{~N}$} \\
\hline Neumayer & $(-70 \mathrm{~N},-8 \mathrm{E})$ & Alert & $(82 \mathrm{~N},-62 \mathrm{E})$ \\
\hline Syowa & $(-69 N, 39 E)$ & Eureka & $(79 \mathrm{~N},-85 \mathrm{E})$ \\
\hline Marambio & $(-64 N,-56 \mathrm{E})$ & Ny-Alesund & $(78 \mathrm{~N}, 11 \mathrm{E})$ \\
\hline Macquarie & $(-54 \mathrm{~N}, 158 \mathrm{E})$ & Resolute & $(74 N,-94 \mathrm{E})$ \\
\hline \multirow[t]{2}{*}{ Broad Meadows } & $(-37 N, 144 \mathrm{E})$ & Lerwick & $(60 \mathrm{~N},-1 \mathrm{E})$ \\
\hline & & Churchill & $(58 \mathrm{~N},-94 \mathrm{E})$ \\
\hline \multirow{2}{*}{\multicolumn{2}{|c|}{ 30S-Eq }} & Edmonton & $(53 \mathrm{~N},-114 \mathrm{E})$ \\
\hline & & Goose Bay & $(53 \mathrm{~N},-60 \mathrm{E})$ \\
\hline Irene & $(-25 \mathrm{~N}, 28 \mathrm{E})$ & Lindenberg & $(52 \mathrm{~N}, 14 \mathrm{E})$ \\
\hline Reunion & $(-21 N, 55 E)$ & deBilt & $(52 \mathrm{~N}, 5 \mathrm{E})$ \\
\hline Fiji & $(-18 \mathrm{~N}, 178 \mathrm{E})$ & Uccle & $(50 \mathrm{~N}, 4 \mathrm{E})$ \\
\hline Samoa & $(-14 \mathrm{~N},-170 \mathrm{E})$ & Praha & $(50 \mathrm{~N}, 14 \mathrm{E})$ \\
\hline Ascension & $(-7 \mathrm{~N},-14 \mathrm{E})$ & Hohenpeissenberg & $(47 N, 11 E)$ \\
\hline Java & $(-7 N, 112 \mathrm{E})$ & Payerne & $(46 \mathrm{~N}, 6 \mathrm{E})$ \\
\hline Natal & $(-5 \mathrm{~N},-35 \mathrm{E})$ & Sapporo & $(43 \mathrm{~N}, 141 \mathrm{E})$ \\
\hline Malindi & $(-2 \mathrm{~N}, 40 \mathrm{E})$ & Trinidad Head & $(40 \mathrm{~N},-124 \mathrm{E})$ \\
\hline Nairobi & $(-1 N, 36 E)$ & Madrid & $(40 \mathrm{~N},-3 \mathrm{E})$ \\
\hline \multirow[t]{2}{*}{ San Cristobal } & $(0 \mathrm{~N},-89 \mathrm{E})$ & Wallops & $(37 N,-75 E)$ \\
\hline & & Tateno & $(36 \mathrm{~N}, 140 \mathrm{E})$ \\
\hline \multirow{2}{*}{\multicolumn{2}{|c|}{$\mathrm{Eq}-30 \mathrm{~N}$}} & Huntsville & $(34 \mathrm{~N},-86 \mathrm{E})$ \\
\hline & & Kagoshima & $(31 \mathrm{~N}, 130 \mathrm{E})$ \\
\hline Sepang & $(2 \mathrm{~N}, 101 \mathrm{E})$ & & \\
\hline Paramaribo & $(5 \mathrm{~N},-55 \mathrm{E})$ & & \\
\hline Hilo & $(19 \mathrm{~N},-155 \mathrm{E})$ & & \\
\hline Hong Kong & $(22 \mathrm{~N}, 114 \mathrm{E})$ & & \\
\hline Naha & $(26 \mathrm{~N}, 127 \mathrm{E})$ & & \\
\hline New Delhi & $(28 \mathrm{~N}, 77 \mathrm{E})$ & & \\
\hline
\end{tabular}

has a positive bias of 7-16 ppbv, indicating the $\mathrm{CO}$ emissions from biomass burning may be over-estimated. The positive bias over Australia is consistent with that seen in the NOAA surface sites, particularly Cape Grim (Fig. 5). Further evaluation of the model can be performed for case studies for which there are more detailed measurements, such as field campaigns.

\subsection{Ozone}

An evaluation of the distribution of ozone in MOZART-4 is made through comparison with ozonesondes, which are available from many sites over the past several decades. The observations used here are from the World Ozone and Ultraviolet Radiation Data Centre (Environment Canada, retrieved 25 March 2009 from http://www.woudc.org). The data originate from many sources, including the SHADOZ network (Thompson et al., 2003). The model-data comparisons are summarized in Fig. 8 for latitude and altitude bins. The mean of all observations, for each month of each year, within each latitude range (90S-30S, 30S-Equator, Equator-30N, 30N$90 \mathrm{~N})$ and within $100 \mathrm{hPa}$ of each altitude used (400, 650, $900 \mathrm{hPa}$ ), are shown as individual points, colored by year. Monthly mean model results have been extracted for each ozonesonde site, and binned as the observations. The sites of the ozonesonde measurements used in each latitude bin are given in Table 11. 

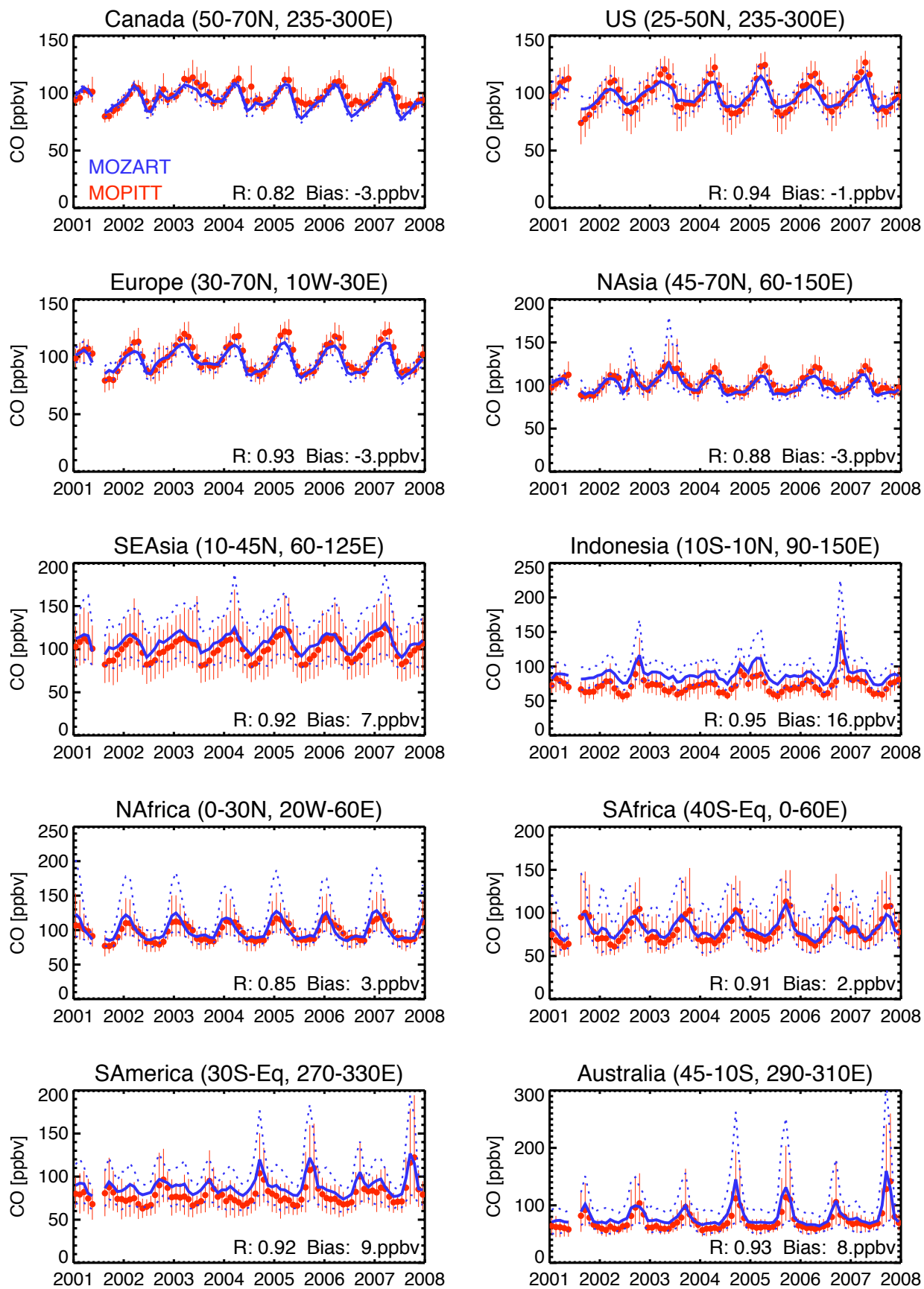

Fig. 7. Comparison of MOZART-4 CO (blue solid and dashed lines) with MOPITT V4 CO (red points and error bars) averaged over regions, based on column averages of monthly means. MOZART-4 results have been transformed with the MOPITT averaging kernels and a priori. The error bars (and dashed lines for MOZART-4) indicate the 10th to 90th percentiles of the range of data over the region for each month.

Generally good agreement is seen between MOZART-4 and the observations (Fig. 8), but some systematic differences are apparent. In the lower troposphere of the Southern Hemisphere, MOZART-4 generally underestimates the ozonesondes, whereas it is slightly high in the Northern Tropics (Eq-30N, 900 and $650 \mathrm{hPa}$ ). Significant interannual variability is seen in the observations in the Southern Tropics in the mid-troposphere (30S-Eq $650 \mathrm{hPa}$ and $400 \mathrm{hPa}$ ) at the end of the year, but MOZART-4 underestimates the concentrations, as well as the variation. MOZART-4 also does not fully reproduce the spring-to-summer increase in ozone in the Northern Hemisphere mid-troposphere (30-90N, 650 and $400 \mathrm{hPa}$ ), most likely due to the constraint on the stratospheric input through the use of SYNOZ, and the low vertical resolution of this simulation with 28 levels. 

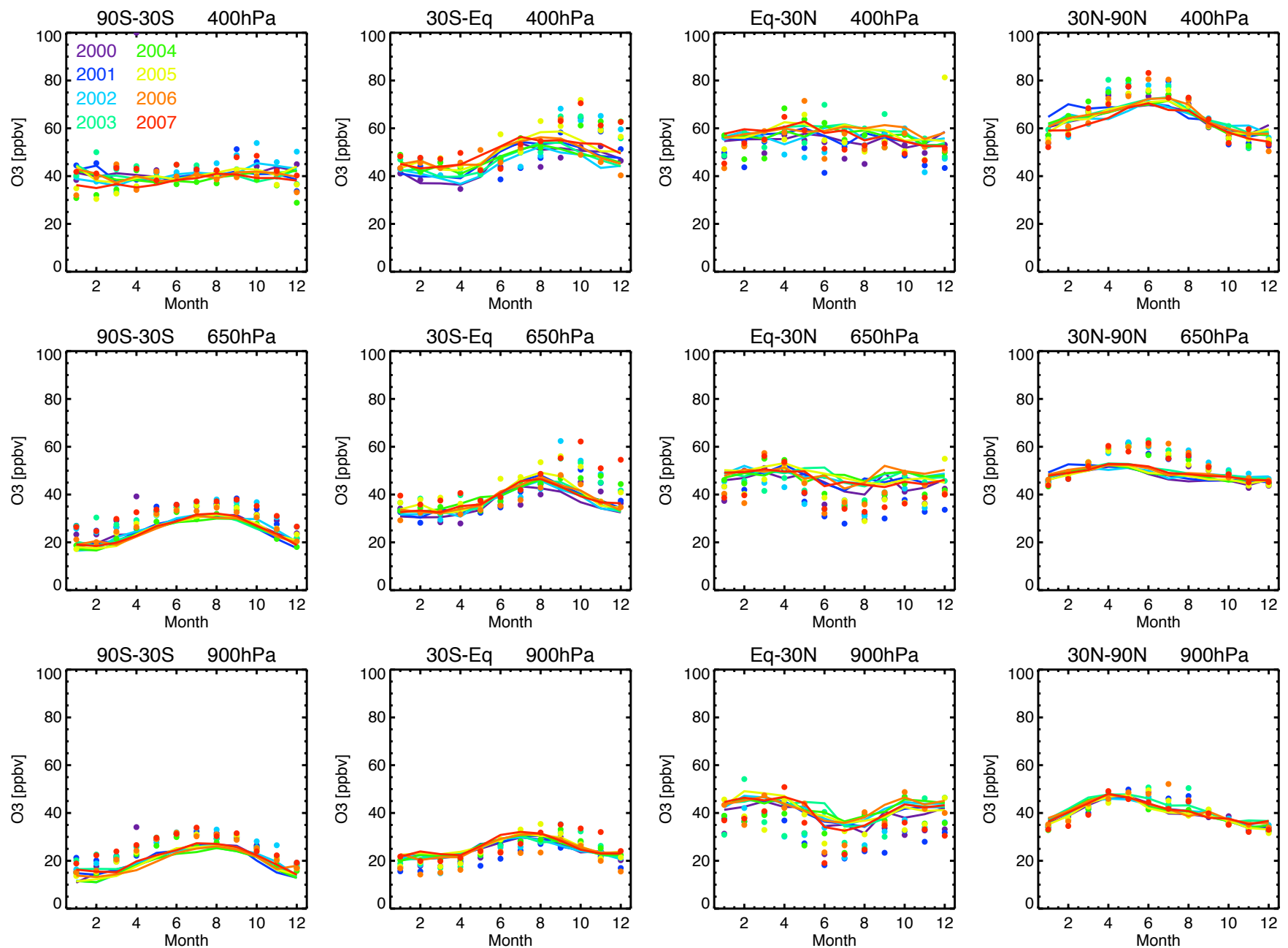

Fig. 8. Evaluation of MOZART-4 with ozonesonde measurements, binned by latitude and altitude, for each year 2000-2007. The number of sites in each region is 90S-30S: 5, 30S-Eq: 10, Eq-30N: 6, 30N-60N: 21. The sites used for each region are given in Table 11. Each dot indicates the average over the month and all sites in the latitude bin, colored by year. The lines indicate MOZART- 4 monthly means, averaged over the ozonesonde locations.

\subsection{Aerosol optical depth}

The aerosol optical depth (AOD) retrievals from MODIS are compared to those calculated by MOZART-4 from the simulated aerosol distributions. Fig. 9 shows averages of MODIS AOD, over oceans only, for various regions, with corresponding averages from MOZART-4. The model agrees very well with the MODIS retrievals over the N. Pacific, which is strongly influenced by the export of pollution from Asia each spring. In May 2003 exceptionally large fires were burning in eastern Russia, resulting in very high AOD values (e.g., Edwards et al., 2004) that the model did not capture. Despite having biomass burning emissions specific for the year (see Sect. 4.1), this indicates the fire emissions inventory underestimated the black carbon or organic carbon emissions. In contrast, the model somewhat over-estimates the AOD over the N. Atlantic Ocean, both in the mean, and in the variability. The AOD from MOZART-4 systematically under-estimates the observations in the Southern Hemisphere. Since the model is too low for all seasons, the washout rates for the aerosols may be too strong in the model. However, the seasonal variation is also under-estimated in these three regions. The annual maxima over the S. Pacific and S. Atlantic Oceans are driven by biomass burning in the second half of the year, with fires in S. America impacting the S. Atlantic, and in Africa and Indonesia impacting the S. Pacific. Therefore, it is likely the Southern Hemisphere biomass burning emissions of aerosols are too low in this simulation. An additional uncertainty in the calculation of AOD lies in the definition of the physical or radiative properties of aerosols, which requires evaluation of the model with simultaneous aerosol size distribution, number density and composition along with radiation measurements. 

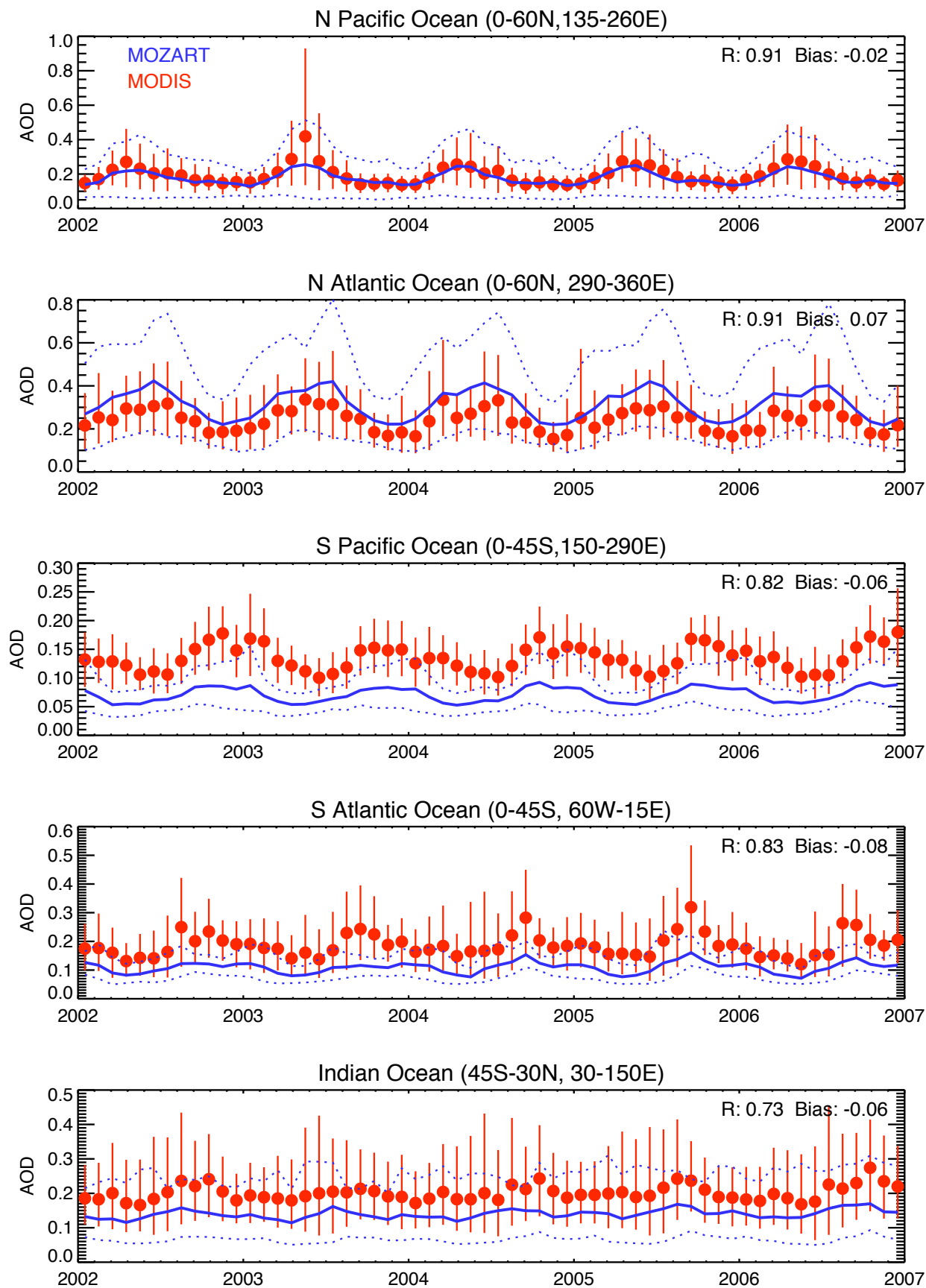

Fig. 9. Evaluation of MOZART-4 AOD with MODIS AOD retrievals. Ocean-only retrievals from MODIS for five regions are compared to ocean-only grid points from MOZART-4 (latitude-longitude ranges indicated in plot titles). Monthly means are shown, with error bars (dotted lines for MOZART-4) indicating the central $80 \%$ of the variation over the region.

\section{Conclusions}

The offline global chemical transport model for the troposphere MOZART-4 includes a number of updates over the previous version MOZART-2, the most significant being the expansion of the chemical mechanism, with the inclusion of aerosols, and the online calculation of photolysis rates, biogenic emissions and dry deposition. Evaluation with several sets of observations shows that MOZART-4 can reproduce well tropospheric chemical composition. When driven with time varying emission inventories (particularly for biomass burning), MOZART-4 reproduces the spatial and temporal variability in observations, such as the NOAA 
GMD network and MOPITT CO, ozonesondes and MODIS aerosol optical depth measurements. MOZART-4 is now available to the community and is suitable for many tropospheric investigations on the regional to global scale. The MOZART-4 source code and standard input files are available for download from the NCAR Community Data Portal (http://cdp.ucar.edu).

Acknowledgements. We acknowledge the many people who have participated in the development and evaluation of all of the versions of the MOZART model over the years, and in particular the leadership of Guy Brasseur. We thank Mary Barth and Valery Yudin for helpful comments on the manuscript. We also acknowledge the science teams producing the data used for model evaluation, including the NOAA Earth System Research Laboratory Global Monitoring Division for surface CO, the Canadian and NCAR MOPITT Teams for MOPITT retrievals, the World Ozone and Ultraviolet Radiation Data Centre (WOUDC) for ozonesondes, and NASA for MODIS AOD retrievals. The National Center for Atmospheric Research is sponsored by the National Science Foundation.

Edited by: V. Grewe

\section{References}

Andreae, M. and Merlet, P.: Emission of trace gases and aerosols from biomass burning, Global Biogeochem. Cy., 15, 966-995, 2001

Andres, R. and Kasgnoc, A.: A time-averaged inventory of subaerial volcanic sulfur emissions, J. Geophys. Res., 103, 2525125261, 1998.

Barth, M. C., Rasch, P. J., Kiehl, J. T., Benkovitz, C. M., and Schwartz, S. E.: Sulfur chemistry in the National Center for Atmospheric Research Community Climate Model: Description, evaluation, features, and sensitivity to aqueous chemistry, J. Geophys. Res., 105, 1387-1415, 2000

Baughcum, S., Tritz, T., Henderson, S., and Pickett, D.: Scheduled Civil Aircraft Emission Inventories for 1992: Database Development and Analysis, NASA Contractor Report 4700, available at: http://ntrs.nasa.gov/archive/nasa/casi.ntrs.nasa.gov/ 19960038445_1996060751.pdf (last access: August 2007), 1996.

Baughcum, S., Henderson, S., and Sutkus, D.: Scheduled Civil Aircraft Emission Inventories Projected for 2015: Database Development and Analysis, NASA/CR-1998-207638, available at: http://ntrs.nasa.gov/archive/nasa/casi.ntrs.nasa.gov/ 19980055200_1998120133.pdf(last access: August 2007), 1998.

Bonan, G., Levis, S., Sitch, S., Vertenstein, M., and Oleson, K.: A dynamic global vegetation model for use with climate models: concepts and description of simulated vegetation dynamics, Glob. Change Biology, 9, 1543-1566, doi:10.1046/j.1529-8817. 2003.00681.x, 2003.

Bond, T., Streets, D. G., Yarber, K. F., Nelson, S. M., Woo, J.H., and Klimont, Z.: A technology-based global inventory of black and organic carbon emissions from combustion, J. Geophys. Res., 109, D14203, doi:10.1029/2003JD003697, 2004.
Bouwman, A. F., Boumans, L. J. M., and Batjes, N. H.: Modeling global annual $\mathrm{N}_{2} \mathrm{O}$ and $\mathrm{NO}$ emissions from fertilized fields, Global Biogeochem. Cy., 16, 1080, doi:10.1029/ 2001GB001812, 2002.

Brasseur, G. P., Hauglustaine, D. A., Walters, S., Rasch, P. J., Muller, J.-F., Granier, C., and Tie, X.-X.: MOZART: A global chemical transport model for ozone and related chemical tracers, Part 1: Model description, J. Geophys. Res., 103, 28265-28289, 1998.

Chang, J. S., Brost, R. A., Isaksen, I. S. A., Madronich, S., Middleton, P., Stockwell, W. R., and Walcek, C. J.: A three-dimensional Eulerian acid deposition model: Physical concepts and model formulation, J. Geophys. Res., 92, 14681-14700, 1987.

Chin, M., Duncan, B. N., Ginoux, P., et al.: Tropospheric aerosol optical thickness from the GOCART model and comparisons with satellite and sun photometer measurements, J. Atmos. Sci., 59, 461-483, 2002.

Chung, S. and Seinfeld, J.: Global distribution and climate forcing of carbonaceous aerosols, J. Geophys. Res., 107, 4407, doi:10. 1029/2001JD001397, 2002.

Cooke, W. F. and Wilson, J. J. N.: A global black carbon aerosol model, J. Geophys. Res., 101, 19395-19409, 1996.

DeCaria, A. J., Pickering, K. E., Stenchikov, G. L., and Ott, L. E.: Lightning-generated NOX and its impact on tropospheric ozone production: A three-dimensional modeling study of a Stratosphere-Troposphere Experiment: Radiation, Aerosols and Ozone (STERAO-A) thunderstorm, J. Geophys. Res., 110, D14303, doi:10.1029/2004JD005556, 2006.

Deeter, M., Edwards, D. P., Gille, J. C., Emmons, L. K., Francis, G., Ho, S.-P., Mao, D., Masters, D., Worden, H., Yudin, V., and Drummond, J. R.: The MOPITT Version 4 CO product: Algorithm enhancements, selected results and bias drift, J. Geophys. Res., doi:10.1029/2009JD013005, in press, 2010.

Dentener, F., Stevenson, D., Ellingsen, K., et al.: Global Atmospheric Environment for the next generation, Environ. Sci. Technol., 40, 3586-3594, 2006.

Dlugokencky, E., Myers, R., Lang, P., Masarie, K., Crotwell, A., Thoning, K., Hall, B., Elkins, J., and Steele, L.: Conversion of NOAA atmospheric dry air methane mole fractions to a gravimetrically-prepared standard scale, J. Geophys. Res., 110, D18306, doi:10.1029/2005JD006035, 2005.

Dlugokencky, E., Lang, P., and Masarie, K.: Atmospheric Methane Dry Air Mole Fractions from the NOAA ESRL Carbon Cycle Cooperative Global Air Sampling Network, 1983-2007, Version: 2008-07-02, available at: ftp://ftp.cmdl.noaa.gov/ccg/ch4/ flask/(last access: August 2008), 2008.

Edwards, D., Emmons, L., Hauglustaine, D., et al.: Observations of carbon monoxide and aerosols from the Terra satellite: Northern Hemisphere variability, J. Geophys. Res., 109, D24202, doi:10. 1029/2004JD004727, 2004.

Emmons, L. K., Edwards, D. P., Deeter, M. N., Gille, J. C., Campos, T., Nédélec, P., Novelli, P., and Sachse, G.: Measurements of Pollution In The Troposphere (MOPITT) validation through 2006, Atmos. Chem. Phys., 9, 1795-1803, 2009, http://www.atmos-chem-phys.net/9/1795/2009/.

Emmons, L. K., Apel, E. C., Lamarque, J.-F., et al.: Impact of Mexico City emissions on regional air quality from MOZART-4 simulations, Atmos. Chem. Phys., submitted, 2010. 
Fast, J., Gustafson Jr., W., Easter, R., Zaveri, R., Barnard, J., Chapman, E., and Grell, G.: Evolution of ozone, particulates, and aerosol direct forcing in an urban area using a new fully-coupled meteorology, chemistry, and aerosol model, J. Geophys. Res., 111, D21305, doi:10.1029/2005JD006721, 2006.

Fast, J. D., de Foy, B., Acevedo Rosas, F., Caetano, E., Carmichael, G., Emmons, L., McKenna, D., Mena, M., Skamarock, W., Tie, X., Coulter, R. L., Barnard, J. C., Wiedinmyer, C., and Madronich, S.: A meteorological overview of the MILAGRO field campaigns, Atmos. Chem. Phys., 7, 2233-2257, 2007, http://www.atmos-chem-phys.net/7/2233/2007/.

Fast, J., Aiken, A. C., Allan, J., Alexander, L., Campos, T., Canagaratna, M. R., Chapman, E., DeCarlo, P. F., de Foy, B., Gaffney, J., de Gouw, J., Doran, J. C., Emmons, L., Hodzic, A., Herndon, S. C., Huey, G., Jayne, J. T., Jimenez, J. L., Kleinman, L., Kuster, W., Marley, N., Russell, L., Ochoa, C., Onasch, T. B., Pekour, M., Song, C., Ulbrich, I. M., Warneke, 'C., Welsh-Bon, D., Wiedinmyer, C., Worsnop, D. R., Yu, X.-Y., and Zaveri, R.: Evaluating simulated primary anthropogenic and biomass burning organic aerosols during MILAGRO: implications for assessing treatments of secondary organic aerosols, Atmos. Chem. Phys., 9, 6191-6215, 2009, http://www.atmos-chem-phys.net/9/6191/2009/.

Gong, S. L., Barrie, L. A., Blanchet, J.-P., von Salzen, K., Lohmann, U., Lesins, G., Spacek, L., Zhang, L. M., Girard, E., Lin, H., Leaitch, R., Leighton, H., Chylek, P., and Huang, P.: Canadian Aerosol Module: A size-segregated simulation of atmospheric aerosol processes for climate and air quality models 1. Module development, J. Geophys. Res., 108, 4007, 10.1029/2001JD002002, 2003.

Granier, C., Guenther, A., Lamarque, J., Mieville, A., Muller, J., Olivier, J., Orlando, J., Peters, J., Petron, G., Tyndall, G., and Wallens, S.: POET, a database of surface emissions of ozone precursors, available at: http://www.aero.jussieu.fr/projet/ ACCENT/POET.php(last access: August 2008), 2005.

Grell, G., Peckham, S., Schmitz, R., McKeen, S., Frost, G., Skamarock, W., and Eder, B.: Fully coupled "online" chemistry within the WRF model, Atmos. Environ., 39, 6957-6976, 2005.

Grewe, V., Reithmeier, C., and Shindell, D.: Dynamic-chemical coupling of the upper troposphere and lower stratosphere region, Chemosphere, 74, 851-861, 2002.

Guenther, A., Karl, T., Harley, P., Wiedinmyer, C., Palmer, P. I., and Geron, C.: Estimates of global terrestrial isoprene emissions using MEGAN (Model of Emissions of Gases and Aerosols from Nature), Atmos. Chem. Phys., 6, 3181-3210, 2006, http://www.atmos-chem-phys.net/6/3181/2006/.

Hack, J. J.: Parameterization of moist convection in the NCAR community climate model (CCM2), J. Geophys. Res., 99, 55515568, 1994.

Hess, M., Koepke, P., and Schult, I.: Optical Properties of Aerosols and Clouds: The software package OPAC, B. Am. Meteorol. Soc., 79, 831-844, 1998.

Holstlag, A. and Boville, B. A.: Local versus nonlocal boundarylayer diffusion in a global climate model, J. Climate, 6, 18251842, 1993.

Horowitz, L., Walters, S., Mauzerall, D., Emmons, L., Rasch, P., Granier, C., Tie, X., Lamarque, J.-F., Schultz, M., Tyndall, G., Orlando, J., and Brasseur, G.: A global simulation of tropospheric ozone and related tracers: Description and evalua- tion of MOZART, version 2, J. Geophys. Res., 108, 4784, doi: 10.1029/2002JD002853, 2003.

Horowitz, L. W.: Past, present, and future concentrations of tropospheric ozone and aerosols: Methodology, ozone evaluation, and sensitivity to aerosol wet removal, J. Geophys. Res., 111, D22211, doi:10.1029/2005JD006937, 2006.

Horowitz, L. W., Fiore, A. M., Milly, G. P., Cohen, R. C., Perring, A., Wooldridge, P. J., Hess, P. G., Emmons, L. K., and Lamarque, J.-F.: Observational constraints on the chemistry of isoprene nitrates over the eastern United States, J. Geophys. Res., 112, D12S08, doi:10.1029/2006JD007747, 2007.

IPCC: Intergovernmental Panel on Climate Change, Emisson Scenarios, A Special Report of IPCC Working Group III, Cambridge University Press, Cambridge, UK, 2000.

Jacob, D. J.: Heterogeneous chemistry and tropospheric ozone, Atmos. Environ., 34, 2131-2159, 2000.

Kalnay, E., Kanamitsu, M., Kistler, R., et al.: The NCEP/NCAR 40Year Reanalysis Project, B. Am. Meteorol. Soc., 77, 437-471, 1996.

Kinnison, D., Brasseur, G. P., Walters, S., et al.: Sensitivity of chemical tracers to meteorological parameters in the MOZART-3 chemical transport model, J. Geophys. Res., 112, D20302, doi: 10.1029/2006JD007879, 2007.

Kistler, R. et al.: The NCEP-NCAR 50-Year Reanalysis: Monthly Means CD-ROM and Documentation, B. Am. Meteorol. Soc., 82, 247-267, 2001.

Kloster, S., Feichter, J., Maier-Reimer, E., Six, K. D., Stier, P., and Wetzel, P.: DMS cycle in the marine ocean-atmosphere system a global model study, Biogeosciences, 3, 29-51, 2006, http://www.biogeosciences.net/3/29/2006/.

Laepple, T., Schultz, M., Lamarque, J.-F., Madronich, S., Shetter, R., Lefer, B., and Atlas, E.: Improved albedo formulation for chemistry transport models based on satellite observations and assimilated snow data and its impact on tropospheric photochemistry, J. Geophys. Res., 110, D11308, doi: 10.1029/2004JD005463, 2005.

Lamarque, J.-F., Kiehl, J. T., Hess, P. G., Collins, W. D., Emmons, L. K., Ginoux, P., Luo, C., and Tie, X. X.: Response of a coupled chemistry-climate model to changes in aerosol emissions: Global impact on the hydrological cycle and the tropospheric burdens of $\mathrm{OH}$, ozone and $\mathrm{NO}_{\mathrm{x}}$, Geophys. Res. Lett., 32, L16809, doi:10.1029/2005GL023419, 2005.

Lapina, K., Honrath, R. E., Owen, R. C., Martín, M. V., and Pfister, G.: Evidence of significant large-scale impacts of boreal fires on ozone levels in the midlatitude Northern Hemisphere free troposphere, Geophys. Res. Lett., 33, L10815, doi: 10.1029/2006GL025878, 2006.

Lawrence, M., Jöckel, P., and von Kuhlmann, R.: What does the global mean $\mathrm{OH}$ concentration tell us?, Atmos. Chem. Phys., 1, 37-49, 2001, http://www.atmos-chem-phys.net/1/37/2001/.

Lawrence, P. J. and Chase, T.: Representing a new MODIS consistent land surface in the Community Land Model (CLM 3.0), J. Geophys. Res., 112, G01023, doi:10.1029/2006JG000168, 2007.

Lin, S. J. and Rood, R. B.: Multidimensional flux-form semiLagrangian transport scheme, Mon. Weather Rev., 124, 20462070, 1996.

Mahowald, N., Lamarque, J.-F., Tie, X., and Wolff, E.: Sea salt aerosol response to climate change: last glacial maximum, pre- 
industrial and doubled carbon dioxide climates, J. Geophys. Res., 111, D05303, doi:10.1029/2005JD006459, 2006a.

Mahowald, N. M., Muhs, D. R., Levis, S., Rasch, P. J., Yoshioka, M., Zender, C. S., and Luo, C.: Change in atmospheric mineral aerosols in response to climate: Last glacial period, preindustrial, modern, and doubled carbon dioxide climates, J. Geophys. Res., 111, D10202, doi:10.1029/2005JD006653, 2006 b.

McLinden, C., Olsen, S., Hannegan, B., Wild, O., and Prather, M.: Stratospheric ozone in 3-D models: A simple chemistry and the cross-tropopause flux, J. Geophys. Res., 105, 14653-14665, 2000.

Mena-Carrasco, M., Carmichael, G. R., Campbell, J. E., Zimmerman, D., Tang, Y., Adhikary, B., D'allura, A., Molina, L. T., Zavala, M., García, A., Flocke, F., Campos, T., Weinheimer, A. J., Shetter, R., Apel, E., Montzka, D. D., Knapp, D. J., and Zheng, W.:Assessing the regional impacts of Mexico City emissions on air quality and chemistry, Atmos. Chem. Phys., 9, 37313743, 2009,

http://www.atmos-chem-phys.net/9/3731/2009/.

Metzger, S., Dentener, F., Pandis, S., and Lelieveld, J.: Gas/aerosol partitioning: 1. A computationally efficient model, J. Geophys. Res., 107(D16), 4312, doi:10.1029/2001JD001102, 2002.

Mortlock, A. and Alstyne, R. V.: Military, Charter, Unreported Domestic Traffic and General Aviation: 1976, 1984, 1992, and 2015 Emission Scenarios, NASA CR-1998-207639, available at: http://ntrs.nasa.gov/archive/nasa/casi.ntrs.nasa.gov/ 19980047346_1998120131.pdf(last access: August 2007), 1998.

Müller, J.-F., Stavrakou, T., Wallens, S., De Smedt, I., Van Roozendael, M., Potosnak, M. J., Rinne, J., Munger, B., Goldstein, A., and Guenther, A. B.: Global isoprene emissions estimated using MEGAN, ECMWF analyses and a detailed canopy environment model, Atmos. Chem. Phys., 8, 1329-1341, 2008,

http://www.atmos-chem-phys.net/8/1329/2008/.

Novelli, P. C., Masarie, K., and Lang, P.: Molecular hydrogen in the troposphere: Global distributions and budget, J. Geophys. Res., 104, 30427-30444, 1999.

Novelli, P. C., Masarie, K., Lang, P., Hall, B., Myers, R., and Elkins, J.: Re-analysis of tropospheric CO trends: Effects of the 1997-1998 wildfires, J. Geophys. Res., 108, 4464, doi: 10.1029/2002JD003031, 2003.

Ohara, T., Akimoto, H., Kurokawa, J., Horii, N., Yamaji, K., Yan, X., and Hayasaka, T.: An Asian emission inventory of anthropogenic emission sources for the period 1980-2020, Atmos. Chem. Phys., 7, 4419-4444, 2007,

http://www.atmos-chem-phys.net/7/4419/2007/.

Olivier, J. and Berdowski, J.: The Climate System, chap. Global emissions sources and sinks, edited by J. Berdowski, R. Guicherit and B.J. Heij, A.A. Balkema Publishers/Swets, Zeitlinger Publishers, Lisse, The Netherlands, 33-78, 2001.

Olivier, J., Peters, J., Granier, C., Petron, G., Müller, J., and Wallens, S.: Present and future surface emissions of atmospheric compounds, POET report \#2, EU project EVK2-1999-00011, available at, http://www.aero.jussieu.fr/projet/ACCENT/POET. php(last access: August 2008), 2003.

Pfister, G., Hess, P., Emmons, L., Lamarque, J.-F., Wiedinmyer, C., Edwards, D., Pétron, G., Gille, J., and Sachse, G.: Quantifying $\mathrm{CO}$ emissions from the 2004 Alaskan wildfires using MOPITT CO data, Geophys. Res. Lett., 32, doi:10.1029/2005GL022995, 2005.
Pfister, G. G., Emmons, L. K., Hess, P. G., Honrath, R., et al.: Ozone production from the 2004 North American boreal fires, J. Geophys. Res., 111, D24S07, doi:10.1029/2006JD007695, 2006.

Pfister, G. G., Emmons, L. K., Hess, P. G., Lamarque, J.-F., Orlando, J. J., Walters, S., Guenther, A., Palmer, P. I., and Lawrence, P. J.: Contribution of isoprene to chemical budgets: A model tracer study with the NCAR CTM MOZART-4, J. Geophys. Res., 113, D05308, doi:10.1029/2007JD008948, 2008a.

Pfister, G. G., Emmons, L. K., Hess, P. G., Lamarque, J.-F., Thompson, A. M., and Yorks, J. E.: Analysis of the Summer 2004 ozone budget over the United States using Intercontinental Transport Experiment Ozonesonde Network Study (IONS) observations and Model of Ozone and Related Tracers (MOZART-4) simulations, J. Geophys. Res., 113, D23306, doi: 10.1029/2008JD010190, 2008b.

Pfister, G. G., Emmons, L. K., Edwards, D. P., et al.: Transpacific pollution transport during INTEX-B: spring 2006 in context to previous years, Atmos. Chem. Phys. Discuss., 9, 17817-17849, 2009, http://www.atmos-chem-phys-discuss.net/9/17817/2009/.

Pickering, K., Wang, Y., Tao, W.-K., Price, C., and Muller, J.-F.: Vertical distributions of lightning $\mathrm{NO}_{\mathrm{x}}$ for use in regional and global transport models, J. Geophys. Res., 103, 31203-31216, 1998.

Price, C. and Rind, D.: A simple lightning parameterization for calculating global lightning distributions, J. Geophys. Res., 97, 9919-9933, doi:10.1029/92JD00719, 1992.

Price, C., Penner, J., and Prather, M.: $\mathrm{NO}_{\mathrm{x}}$ from lightning 1. Global distribution based on lightning physics, J. Geophys. Res., 102, 5929-5941, 1997.

Rasch, P. J., Mahowald, N. M., and Eaton, B. E.: Representations of transport, convection, and the hydrologic cycle in chemical transport models: Implications for the modeling of short-lived and soluble species, J. Geophys. Res., 102, 28127-28138, 1997.

Ridley, B., Pickering, K., and Dye, J.: Comments on the parameterization of lightning-produced $\mathrm{NO}$ in global chemistry-transport models, Atmos. Environ., 39, 6184-6187, 2005.

Sander, S., Friedl, R. R., Ravishankara, A. R., et al.: Chemical Kinetics and Photochemical Data for Use in Atmospheric Studies, Evaluation Number 14, JPL Publication 02-25, NASA, Jet Propulsion Laboratory, California Institute of Technology, Pasadena, CA, 2003.

Sanderson, M., Collins, W., Derwent, R., and Johnson, C.: Simulation of global hydrogen levels using a lagrangian threedimensional model, J. Atmos. Chem., 46, 15-28, 2003.

Seinfeld, J. H.: Atmospheric Chemistry and Physics of Air Pollution, Wiley, New York, 1986.

Shindell, D. T., Faluvegi, G., Stevenson, D., et al.: Multimodel simulations of carbon monoxide: Comparison with observations and projected near-future changes, J. Geophys. Res., 111, D19306, doi:10.1029/2006JD007100, 2006.

Simmons, A. and Gibson, J.: The ERA-40 project plan, in ERA40 Project Report Series, Tech. Rep. 1, Eur. Cent. For MediumRange Weather Forecasts, Reading, UK, 2000.

Singh, H. B., Brune, W. H., Crawford, J. H., Flocke, F., and Jacob, D. J.: Chemistry and transport of pollution over the Gulf of Mexico and the Pacific: spring 2006 INTEX-B campaign overview and first results, Atmos. Chem. Phys., 9, 2301-2318, 2009, http://www.atmos-chem-phys.net/9/2301/2009/. 
SPARC: Report No. 4, Assessment of Stratospheric Aerosol Properties, WCRP-124, WMO/TD-No 1295, 2006.

Sparks, J. P., Roberts, J. M., and Monson, R. K.: The uptake of gaseous organic nitrogen by leaves: A significant global nitrogen transfer process, Geophys. Res. Lett., 30, 2189, doi: 10.1029/2003GL018578, 2003.

Spivakovsky, C. M., Logan, J. A., Montzka, S. A., Balkanski, Y. J., et al.: Three-dimensional climatological distribution of tropospheric OH: Update and evaluation, J. Geophys. Res., 105, 89318980, 2000.

Stevenson, D. S., Dentener, F., Schultz, M., et al.: Multimodel ensemble simulations of present-day and near-future tropospheric ozone, J. Geophys. Res., 111, D08301, doi:10.1029/ 2005JD006338, 2006.

Sutkus, D., Baughcum, S., and DuBois, D.: Scheduled Civil Aircraft Emission Inventories for 1999: Database Development and Analysis, NASA/CR-2001-211216, available at: http://gltrs.grc. nasa.gov/reports/2001/CR-2001-211216.pdf(last access: August 2007), 2001.

Tang, I. N. and Munkelwitz, H. R.: Water activities, densities and refractive indices of aqueous sulfates and sodium nitrate droplets of atmospheric importance, J. Geophys. Res., 99, 18801-18808, 1994.

Tang, Y., Lee, P., Tsidulko, M., et al.: The impact of chemical lateral boundary conditions on CMAQ predictions of tropospheric ozone over the continental United States, Environ. Fluid Mech., 9, 43-58, doi:10.1007/s10652-008-9092-5, 2009.

Tang, Y. H., Carmichael, G. R., Thongboonchoo, N., Chai, T. F., Horowitz, L. W., Pierce, R., Al-Saadi, J. A., Pfister, G., Vukovich, J. M., Avery, M. A., Sachse, G. W., Ryerson, T. B., Holloway, J. S., Atlas, E. L., Flocke, F. M., Weber, R. J., Huey, L. G., Dibb, J. E., Streets, D., and Brune, W. H.: Influence of lateral and top boundary conditions on regional air quality prediction: A multiscale study coupling regional and global chemical transport models, J. Geophys. Res., 112, D10S18, doi: 10.1029/2006JD007515, 2007.

Teklemariam, T. and Sparks, J.: Gaseous fluxes of peroxyacetyl nitrate (PAN) into plant leaves, Plant Cell Envrion., 27, 11491158, 2004

Thompson, A., Witte, J., McPeters, R., Oltmans, S., et al.: Southern Hemisphere Additional Ozonesondes (SHADOZ) 1998-2000 tropical ozone climatology 1. Comparison with Total Ozone Mapping Spectrometer (TOMS) and ground-based measurements, J. Geophys. Res., 108, 8238, doi:10.1029/2001JD000967, 2003.

Tie, X., Brasseur, G., Emmons, L., Horowitz, L., and Kinnision, D.: Effects of aerosols on tropospheric oxidants: A global model study, J. Geophys. Res., 106, 2931-2964, 2001.
Tie, X., Madronich, S., Walters, S., Rasch, P., and Collins, W.: Effect of clouds on photolysis and oxidants in the troposphere, J. Geophys. Res., 108, 4642, doi:10.1029/2003JD003659, 2003.

Tie, X., Madronich, S., Walters, S., Edwards, D., Ginoux, P., Mahowald, N., Zhang, R., Luo, C., and Brasseur, G.: Assessment of the global impact of aerosols on tropospheric oxidants, J. Geophys. Res., 110, D03204, doi:10.1029/2004JD005359, 2005.

Toon, O. B., Pollack, J. B., and Khare, B. N.: The optical constants of several atmospheric aerosol species: ammonium sulfate, aluminum oxide and sodium chloride, J. Geophys. Res., 81, 57335744, 1976.

Turnipseed, A., Huey, G., Nemitz, E., Stickel, R., Higgs, J., Tanner, D. and Slusher, D., Sparks, J., Flocke, F., and Guenther, A.: Eddy covariance fluxes of peroxyacyl nitrates (PANs) and $\mathrm{NO}_{\mathrm{y}}$ to a coniferous forest, J. Geophys. Res., 111, D09304, doi:10.1029/ 2005JD006631, 2006.

van der Werf, G. R., Randerson, J. T., Giglio, L., Collatz, G. J., Kasibhatla, P. S., and Arellano Jr., A. F.: Interannual variability in global biomass burning emissions from 1997 to 2004, Atmos. Chem. Phys., 6, 3423-3441, 2006, http://www.atmos-chem-phys.net/6/3423/2006/.

Van Noije, T., Eskes, H., van Weele, M., and van Velthoven, P.: Implications of the enhanced Brewer-Dobson circulation in European Centre for Medium-Range Weather Forecasts reanalysis ERA-40 for the stratosphere-troposphere exchange of ozone in global chemistry transport models, J. Geophys. Res., 109, D19308, doi:10.1029/2004JD004586, 2004.

Walmsley, J. and Wesely, M.: Modification of coded parametrizations of surface resistances to gaseous dry deposition, Atmos. Environ., 30, 1181-1188, 1996.

Wesely, M.: Parameterization of surface resistances to gaseous dry deposition in regional-scale numerical models, Atmos. Environ., 23, 1293-1304, 1989.

Wesely, M. and Hicks, B.: A review of the current status of knowledge on dry deposition, Atmos. Environ., 34, 2261-2282, 2000.

Yienger, J. and Levy, H.: Empirical model of global soil biogenic $\mathrm{NO}_{\mathrm{x}}$ emissions, J. Geophys. Res., 100, 11447-11464, 1995.

Yonemura, S., Kawashima, S., and Tsuruta, H.: Carbon monoxide, hydrogen, and methane uptake by soils in a temperate arable field and a forest, J. Geophys. Res., 105, 14347-14362, 2000.

Zender, C. S., Bian, H., and Newman, D.: Mineral Dust Entrainment and Deposition (DEAD) model: Description and 1990s dust climatology, J. Geophys. Res., 108, 4416, doi:10.1029/ 2002JD002775, 2003.

Zhang, G. J. and MacFarlane, N. A.: Sensitivity of climate simulations to the parameterization of cumulus convection in the Canadian climate centre general circulation model, Atmos. Ocean, 33 , 407-446, 1995. 